\title{
The Cost of Legal Restrictions on Experience Rating
}

\author{
Levon Barseghyan \\ Cornell University \\ Francesca Molinari \\ Cornell University \\ Darcy Steeg Morris \\ U.S. Census Bureau \\ Joshua C. Teitelbaum \\ Georgetown University Law Center, jct48@law.georgetown.edu
}

Georgetown Business, Economics and Regulatory Law Research Paper No. 12-040

This paper can be downloaded free of charge from:

https://scholarship.law.georgetown.edu/facpub/1127

http://ssrn.com/abstract=2176295

Levon Barseghyan, Francesca Molinari, Darcy Steeg Morris \& Joshua C. Teitelbaum, The Cost of Legal Restrictions on Experience Rating, 17 J. Empirical Legal Stud. 38 (2020).

This open-access article is brought to you by the Georgetown Law Library. Posted with permission of the author. Follow this and additional works at: https://scholarship.law.georgetown.edu/facpub

Part of the Insurance Law Commons, and the Law and Economics Commons 


\title{
The Cost of Legal Restrictions on Experience Rating
}

\author{
Levon Barseghyan, Francesca Molinari, Darcy Steeg Morris, and Joshua C. Teitelbaum*
}

\begin{abstract}
We investigate the cost of legal restrictions on experience rating in auto and home insurance. The cost is an opportunity cost as experience rating can mitigate the problems associated with unobserved heterogeneity in claim risk, including mispriced coverage and resulting demand distortions. We assess this cost through a counterfactual analysis in which we explore how risk predictions, premiums, and demand in home insurance and two lines of auto insurance would respond to unrestricted multiline experience rating. Using claims data from a large sample of households, we first estimate the variance-covariance matrix of unobserved heterogeneity in claim risk. We then show that conditioning on claims experience leads to material refinements of predicted claim rates. Lastly, we assess how the households' demand for coverage would respond to multiline experience rating. We find that the demand response would be large.
\end{abstract}

${ }^{*}$ Address correspondence to Joshua C. Teitelbaum, Georgetown University Law Center, 600 New Jersey Avenue NW, Washington, DC 20001; email: jct48@law.georgetown.edu. Barseghyan is Professor of Economics, Cornell University; Molinari is H. T. Warshow and Robert Irving Warshow Professor of Economics and Professor of Statistics, Cornell University; Morris is Research Mathematical Statistician, Center for Statistical Research and Methodology, U.S. Census Bureau; Teitelbaum is Agnes N. Williams Research Professor and Professor of Law, Georgetown University Law Center. We thank the editor and the referees for their helpful comments and feedback. We acknowledge financial support from National Science Foundation grant SES-1031136. Molinari also acknowledges financial support from NSF grant SES-0922330. This paper is released to inform interested parties of research and to encourage discussion. The views expressed are those of the authors and not necessarily those of the U.S. Census Bureau. 


\section{INTRODUCTION}

In many insurance markets, there are variables that affect an insured's claim risk but are not observable by the insurer. ${ }^{1}$ In other words, there is unobserved heterogeneity in claim risk. The problem with unobserved heterogeneity in claim risk is that it can lead to mispriced insurance, which in turn can impair the efficient operation of insurance markets, including by distorting the demand for insurance coverage.

In theory, an insurer can mitigate these problems through experience rating. The logic is straightforward. Even if there is unobserved heterogeneity in claim risk at the time the insurer underwrites and rates an insured's policy, the insurer subsequently receives signals about the insured's latent risk type. In particular, the insurer observes the insured's claims experience. By conditioning on the insured's claims experience, the insurer can refine its initial prediction about the insured's claim risk, which is based solely on observables. The insurer can then use its refined prediction to adjust - or experience rate - the insured's premium to better reflect her true claim risk. ${ }^{2}$

In practice, however, U.S. law frequently imposes restrictions on an insurer's ability to engage in experience rating. ${ }^{3}$ An example from federal law is the Affordable Care Act's community rating provisions, which forbid experience rating of premiums for heath insurance coverage offered in the individual or small group market. ${ }^{4}$ A state law example is New York's Insurance Law, which forbids experience rating of premiums for auto comprehensive or home

\footnotetext{
${ }^{1}$ Alternatively, there may be variables that are observable by the insurer but that the insurer is prohibited from using when it underwrites or rates the insured's policy (Salani 1997; Avraham et al. 2014).

${ }^{2}$ Experience rating is not to be confused with classification rating. Under classification rating, an insured's premium is based on the collective loss experience of all insureds in the insured's risk class. Under experience rating, by contrast, an insured's premium is adjusted based on her individual loss experience.

${ }^{3}$ Advocates for legal restrictions on experience rating (and other forms of risk classification) generally rely on arguments from equity (distributional and deontological) (e.g., Abraham 1985; Avraham et al. 2014). For instance, they argue that such restrictions promote access to insurance for high-risk, low-income insureds (e.g., Meier 1991; Thiery \& Van Schoubroeck 2006; Thomas 2007; Dionne \& Rothschild 2014). That said, many consider efficiency questions as well (e.g., Abraham 1985; Avraham et al. 2014; Dionne \& Rothschild 2014; Abraham \& Chiappori 2015).

${ }^{4}$ See Patient Protection and Affordable Care Act $\S 2701,42$ U.S.C. $\S 300 g g$ (2018).
} 
insurance coverage and also prohibits using auto comprehensive claims to experience rate premiums in any other line of insurance coverage. ${ }^{5}$

In this paper, we empirically investigate the cost of legal restrictions on experience rating in the context of auto and home insurance. The cost is an opportunity cost. As noted above, experience rating has the potential to mitigate the problems associated with unobserved heterogeneity in claim risk. When the law imposes restrictions on experience rating, insurers lose the opportunity to fully utilize their insureds' claims experience to refine their risk predictions and adjust their premiums to better reflect the true risks. We assess this opportunity cost through a counterfactual analysis in which we explore how risk predictions, premiums, and demand in two lines of auto coverage and one line of home coverage would respond to unrestricted experience rating within and across the three lines of coverage.

Our data comprise an unbalanced panel of 62,425 households who purchased auto and home policies from a single insurance company between 1998 and 2006. Among other things, the data record the number of claims filed by each household in three lines of coverage: auto collision, auto comprehensive, and home all perils. In addition, the data contain detailed information about the households and their auto and home policies.

Our analysis proceeds in three steps. First, we use the data to estimate the variancecovariance matrix $\boldsymbol{\Sigma}$ of unobserved heterogeneity in claim risk and to generate the households' predicted claim rates based on observables. We model households' claim counts using a Poisson mixture model with correlated random effects. To estimate the model, we take a moments-based approach that uses generalized estimating equations based on marginal moments (Morris 2012). Unlike the standard approach - maximum likelihood estimation of a parametric mixture of Poisson distributions - our estimation approach is semiparametric and unconstrained with respect to the parameters of the mixing distribution (Pinquet 2013). Among other things, the estimates reveal that unobserved heterogeneity in claim risk is positively correlated across lines of coverage.

\footnotetext{
${ }^{5}$ See N.Y. Ins. Law $\S 2334$ (2018); N.Y. Comp. Codes R. \& Regis. tit. 11, $\S 161.8$ \& 169.1 (2018).
} 
Next, we demonstrate the value of the information contained in $\widehat{\Sigma}$-and, by implication, the value of the signals provided by the households' claims histories - by showing that conditioning on claims experience leads to material refinements of the households' predicted claim rates. For instance, we find that (i) among households with downward revisions, their predicted claim rates decrease on average by 7 percent in auto collision, 13 percent in auto comprehensive, and 14 percent in home and (ii) among households with upward revisions, their predicted claim rates increase on average by 10 percent in auto collision, 23 percent in auto comprehensive, and 28 percent in home. We also demonstrate the incremental value of conditioning across lines of coverage (in addition to conditioning within lines of coverage).

Finally, we investigate the extent to which the households' demand for coverage, as captured by their deductible choices, would respond to experience rating within and across lines of coverage (i.e., uniline and multiline experience rating). In so doing, we obtain a lower bound on the potential for unpriced heterogeneity in claim risk to distort demand. Our experience rating scheme is a simple bonus-malus system under which changes in premiums are proportional to changes in predicted claim risk. We model households' deductible choices according to standard expected utility theory. After calibrating the model with the risk aversion estimate reported by Barseghyan et al. (2013), we use the model to generate deductible choices for the households in our data assuming first that premiums are not experience rated and then that they are experience rated. We find that there would be large responses to experience rating. In particular, we find that the fraction of households that would change deductibles if premiums were experience rated is 7 percent in auto collision, 21 percent in auto comprehensive, and 15 percent in home, resulting in average changes in coverage of $\$ 247, \$ 178$, and $\$ 347$, respectively, among policies with a change.

The paper proceeds as follows. Section II discusses the related literature. Section III describes our data. Section IV presents the model and explains our estimation approach. Sections V-VII contain the three steps of our analysis. Section VIII offers concluding remarks. 


\section{RelATED Literature}

The paper contributes to two literatures. The first is the literature on experience rating in insurance markets. For surveys, see, e.g., Pinquet $(2000,2013)$ and Antonio \& Valdez (2012). ${ }^{6}$ Most closely related are the handful of papers on multiline experience rating, beginning with Jewell (1974). For example, Pinquet (1998) studies experience rating across auto claims at fault and not at fault; Desjardins et al. (2001) and Angers et al. (2006) study experience rating for fleets of vehicles; Frees (2003) studies experience rating across multiple lines within auto insurance; Englund et al. (2008) and Englund et al. (2009) study experience rating across various types of commercial coverage; Frees et al. (2010) study experience rating across multiple perils within home insurance; and Antonio et al. (2011) study experience rating across multiple auto insurance policies. There are two main differences between these papers and ours. First, we study experience rating across home insurance (all perils) and two lines of auto insurance (collision and comprehensive). ${ }^{7}$ Second, and more importantly, we also study the effects of multiline experience rating on the demand for coverage. This underscores a key difference in focus. Whereas these studies focus on the actuarial science of experience rating, we focus on the economics of legal restrictions on experience rating.

The second related literature is the empirical literature on the regulation of insurance markets, and in particular the strand that seeks to quantify the economic effects of legal restrictions on risk classification by insurers. For example, Buchmueller \& DiNardo (2002), Simon (2005), and Bundorf \& Simon (2006) study the effects of community rating in U.S. health insurance markets; Finkelstein et al. (2009) study the effects of a ban on gender-based pricing in the U.K. annuity market; and Bundorf et al. (2012) and Geruso (2017) study the effects of uniform contribution requirements in the U.S. employer-provided health insurance market. ${ }^{8}$ Again, there are the two main differences between these papers and ours. First,

\footnotetext{
${ }^{6}$ For textbook treatments, see Lemaire (1995), Bhlmann \& Gisler (2005), and Denuit et al. (2007).

${ }^{7}$ Thuring (2011) and Thuring et al. (2012) study auto and home insurance but with a focus on cross-selling.

${ }^{8}$ Though it is not their focus, Einav et al. (2010) also consider the effects of legal restrictions on risk classification in the U.S. employer provided health insurance market. There also is a rich theoretical literature
} 
we study the regulation of the U.S. auto and home insurance markets. Second, whereas these papers study the effects of restrictions on ex ante risk classification (i.e., classification rating), we study the effects of restrictions on ex post risk classification (i.e., experience rating).

\section{Description of the DAtA}

The source of the data is a large U.S. property and casualty insurance company. The company offers auto and home insurance. The full data set includes annual information on more than 400,000 households who purchased auto or home policies from the company between 1998 and 2006. All of the policies in the data are governed by New York law. ${ }^{9}$ The data contain all the information in the company's records regarding the households and their policies. In addition, the data record the number of claims that each household filed with the company under each of its policies during the period of observation.

We focus our attention on three lines of coverage: auto collision, auto comprehensive, and home all perils. Auto collision coverage pays for damage to the insured vehicle caused by a collision with another vehicle or object, without regard to fault. Auto comprehensive coverage pays for damage to the insured vehicle from all other causes (e.g., theft, fire, flood, windstorm, glass breakage, vandalism, hitting or being hit by an animal or by falling or flying objects), without regard to fault. Home all perils coverage pays for damage to the insured home from all causes (e.g., fire, windstorm, hail, tornadoes, vandalism, or smoke damage), except those that are specifically excluded (e.g., flood, earthquake, or war). For simplicity, we often refer to home all perils merely as home.

on insurance regulation and the social welfare implications of legal restrictions on risk classification (e.g., Hoy 1982; Crocker \& Snow 1986; Hoy 2006; Thomas 2008; Crocker \& Snow 2011; Rothschild 2011).

${ }^{9}$ As noted above, New York forbids experience rating of premiums for auto comprehensive or home insurance coverage and also prohibits using auto comprehensive claims to experience rate premiums in any other line of insurance coverage. See N.Y. Ins. Law $\S 2334$ (2018); N.Y. Comp. Codes R. \& Regis. tit. 11, $\S \S 161.8 \& 169.1$ (2018). 
In most of the analysis, we consider an unbalanced panel of 62,425 households who purchased all three coverages (auto collision, auto comprehensive, and home) in one or more years between 1998 and 2006. In all, this tricoverage sample comprises 294,917 householdyears. Table A1 in the Appendix provides descriptive statistics for the tricoverage sample. Tables A2, A3, and A4 in the Appendix summarize the claims, premiums, and deductibles in the tricoverage sample. The mean number of claims per household-year is 0.107 in auto collision, 0.032 in auto comprehensive, and 0.079 in home. ${ }^{10}$ On average, households paid annual premiums of $\$ 200$ in auto collision, $\$ 127$ in auto comprehensive, and $\$ 548$ in home. The mean deductibles per household-year are $\$ 396, \$ 273$, and $\$ 350$ in auto collision, auto comprehensive, and home, respectively. The modal deductibles are $\$ 500$ in auto collision, $\$ 200$ in auto comprehensive, and $\$ 250$ in home.

\section{Model and Estimation Approach}

A standard regression model for longitudinal univariate count data is the Poisson random effects model. We extend this model to multivariate count data-here, claim counts under three types of insurance coverage - by allowing for correlated random effects. ${ }^{11}$

Let $y_{i t k}$ denote the number of claims for household $i$ in year $t$ under coverage $k$, where $i=1, \ldots, N, t=1, \ldots, T_{i}$, and $k \in\{c, m, h\}$. In the set of coverages, $c$ denotes auto collision, $m$ denotes auto comprehensive, and $h$ denotes home. Similarly, let $\mathbf{x}_{i t k}$ denote a vector of observables (plus a constant) for household $i$ in year $t$ under coverage $k \cdot{ }^{12}$ Let $\lambda_{i t k}$ denote

\footnotetext{
${ }^{10}$ In the data, we observe the frequency of claims but not their severity. Hence, we focus on and define claim risk in terms of claim frequency. In our model of deductible choice, we assume that the loss associated with every claim exceeds the maximum deductible option; see Section VII.

${ }^{11}$ By allowing for random effects, our model accounts for overdispersion, including due to excess zeros, in a similar way as the (pooled) negative binomial model (see, e.g., Wooldridge 2002, ch. 19). An alternative approach would be a zero-inflated model. However, Vuong (1989) and likelihood ratio tests select the negative binomial model over the zero-inflated model, suggesting that adjustment for excess zeros is not necessary once we allow for random effects.

${ }^{12}$ The variables that comprise $\mathbf{x}_{i t k}$ are listed in Tables A5 (auto) and A6 (home) in the Appendix. In auto, they include the age, gender, and insurance score (which is based on information contained in credit reports) of the primary driver, the age and gender of each additional driver, and the age, use, location, and safety features of each vehicle. In home, they include the insurance score of the primary owner, the age,
} 
household $i$ 's baseline claim rate in year $t$ under coverage $k$, and let $\epsilon_{i k}$ denote a time-constant random effect for household $i$ under coverage $k$. Both $\lambda_{i t k}$ and $\epsilon_{i k}$ are unobserved.

We assume that claims for household $i$ in year $t$ under coverage $k$ follow a Poisson arrival process with rate $\lambda_{i t k} \epsilon_{i k}$. Specifically, we assume

$$
y_{i t k} \mid \mathbf{x}_{i t k} \sim \text { Poisson }\left(\lambda_{i t k} \epsilon_{i k}\right)
$$

where

$$
\lambda_{i t k}=\exp \left(\mathbf{x}_{i t k}^{\prime} \beta_{k}\right)
$$

and $\epsilon_{i} \equiv\left[\epsilon_{i c} \epsilon_{i m} \epsilon_{i h}\right]^{\prime}$ is iid with $E\left(\epsilon_{i}\right)=\left[\begin{array}{lll}1 & 1 & 1\end{array}\right]^{\prime}$ and $V\left(\epsilon_{i}\right)=\Sigma$.

The parameters to be estimated are

$$
\beta \equiv\left[\begin{array}{c}
\beta_{c} \\
\beta_{m} \\
\beta_{h}
\end{array}\right] \text { and } \boldsymbol{\Sigma} \equiv\left[\begin{array}{ccc}
\sigma_{c}^{2} & \rho_{m c} \sigma_{m} \sigma_{c} & \rho_{h c} \sigma_{h} \sigma_{c} \\
\rho_{c m} \sigma_{c} \sigma_{m} & \sigma_{m}^{2} & \rho_{h m} \sigma_{h} \sigma_{m} \\
\rho_{c h} \sigma_{c} \sigma_{h} & \rho_{m h} \sigma_{m} \sigma_{h} & \sigma_{h}^{2}
\end{array}\right]
$$

Of principal interest is the variance-covariance matrix, $\boldsymbol{\Sigma}$, which captures both the withincoverage variance of unobserved heterogeneity in claim risk, $\sigma^{2} \equiv\left(\sigma_{c}^{2}, \sigma_{m}^{2}, \sigma_{h}^{2}\right)$, and its crosscoverage correlation structure, $\rho \equiv\left(\rho_{c m}, \rho_{c h}, \rho_{m h}\right)$.

The likelihood function may be written as

$$
\mathcal{L}_{i}=\int_{\epsilon_{i h}} \int_{\epsilon_{i m}} \int_{\epsilon_{i c}}\left\{\prod_{k} \prod_{t} \exp \left(-\lambda_{i t k} \epsilon_{i k}\right) \frac{\left(\lambda_{i t k} \epsilon_{i k}\right)^{y_{i t k}}}{y_{i t k} !}\right\} f\left(\epsilon_{i c}, \epsilon_{i m}, \epsilon_{i h}\right) d \epsilon_{i c} d \epsilon_{i m} d \epsilon_{i h}
$$

where $f\left(\epsilon_{i c}, \epsilon_{i m}, \epsilon_{i h}\right)$ is the trivariate density of $\epsilon_{i}$. A standard parametric approach is to specify $f$ and estimate the model by maximum likelihood. Typical specifications of $f$ include the lognormal distribution and the gamma distribution (in which case $\mathcal{L}_{i}$ reduces to the product of negative binomial densities). In our case, however, the standard approach is value, use, location, type of construction, and safety features of the dwelling, whether the dwelling is owner occupied, and the number of families that occupy the dwelling. 
computationally intractable. The likelihood function not only involves a multidimensional integral, but, depending on $f$, it also may not have a closed-form expression.

We adopt a semiparametric, moments-based approach, which provides a computationally tractable method for consistent estimation of $\beta$ and $\boldsymbol{\Sigma}$ for all possible densities $f$. Under this approach, estimation is via generalized estimating equations (GEE) based on marginal moments. ${ }^{13}$ Given the assumptions of our model, we can derive the first and second marginal moments and use them to construct estimating equations for $\beta$ and $\boldsymbol{\Sigma}$. More specifically, we use the first marginal moment to define a quasi-score equation, where the associated estimating equation for $\beta$ is based on a weighted least squares estimator with the weight matrix defined by the covariance structure derived from the second marginal moment. The estimating equation for $\boldsymbol{\Sigma}$ is based on the relation between the empirical variance estimate and the model defined covariance structure. The two estimating equations are solved iteratively to obtain $\widehat{\beta}$ and $\widehat{\Sigma}$. For further details about the estimation approach, see the Appendix. ${ }^{14}$

\section{Estimation Results}

\section{A. Regression Estimates}

Table 1 presents the estimates of the association parameters, $\sigma^{2}$ and $\rho$, implied by $\widehat{\Sigma}$. The estimates reveal that the variance of unobserved heterogeneity is lowest in auto collision $\left(\widehat{\sigma}_{c}^{2}=0.11\right)$ and is roughly four times higher in auto comprehensive $\left(\widehat{\sigma}_{m}^{2}=0.40\right)$ and home $\left(\widehat{\sigma}_{h}^{2}=0.41\right)$. More importantly, the estimates also reveal that unobserved heterogeneity is correlated across coverages - each pairwise correlation is positive and statistically signifi-

\footnotetext{
${ }^{13} \mathrm{GEE}$ were introduced by Liang and Zeger and co-authors in the 1980s (see, e.g., Liang \& Zeger 1986; Zeger \& Liang 1986; Zeger et al. 1988). For a textbook treatment of GEE, see, e.g., Ziegler (2011).

${ }^{14}$ See also Morris (2012) and Pinquet (2013). This approach is an extension of quasi-generalized pseudo maximum likelihood (QGPML) estimators developed by Gouriroux et al. (1984a,b) and the extended GEE approach developed by Prentice (1988). The QGPML method can be characterized as first order GEE with a specific association structure. Prentice introduced an extension of first order GEE that utilizes a second set of estimating equations to jointly estimate the association parameters. QGPML can be embedded in the GEE framework resulting in commonly studied consistency and asymptotic results for simultaneous inference on both the regression parameters and the association parameters.
} 
cant. Perhaps not surprisingly, the strongest correlation is between auto collision and auto comprehensive $\left(\widehat{\rho}_{c m}=0.66\right)$. There is also a fairly strong correlation between auto comprehensive and home $\left(\widehat{\rho}_{m h}=0.56\right)$. The weakest correlation is between auto collision and home $\left(\widehat{\rho}_{c h}=0.29\right)$. Even this correlation, however, is economically significant, as we demonstrate in Sections VI and VII.

Our primary interest in these correlations is their instrumental value in terms of predicting claim risk and experience rating, which we explore in Sections VI and VII. However, they are also interesting in their own right, because they suggest that, even after controlling for observable characteristics, there exists a latent, domain-general component to risk type. We elaborate on this point in our concluding discussion in Section VIII.

The estimates of the regression parameters, $\beta$, are reported in Tables A5 (auto) and A6 (home) in the Appendix. Because $\beta$ is not the object of primary interest, we relegate our comments about the regression parameter estimates to the Appendix as well.

\section{B. Robustness Checks}

\section{Alternative Samples}

As a check of the sensitivity of the association parameter estimates to our sample restrictions, we re-estimate the model on two alternative samples of the data: (A) a balanced panel of 8,731 households $(78,579$ household-years) who purchased all three coverages (auto collision, auto comprehensive, and home); and (B) an unbalanced panel of 203,731 households (1,019,170 household-years) who purchased both auto coverages (collision and comprehensive). The association parameter estimates for both alternative samples are reported in Table A7 in the Appendix. ${ }^{15}$ They are largely consistent with the estimates for the tricoverage sample. If anything, they suggest that our baseline estimates are conservative.

\footnotetext{
${ }^{15}$ To ease the computational burden of the re-estimations, we obtain estimates of the regression parameters from a generalized linear model (GLM) assuming the random effects follow a lognormal distribution. In the tricoverage sample, the semiparametric and GLM estimates for $\beta$ are nearly identical $\left(R^{2}=0.9998\right)$. Thus, we are confident that using the GLM estimates for $\beta$ does not corrupt the semiparametric estimates of the association parameters in the re-estimations.
} 


\section{Moral Hazard}

Our approach implicitly assumes that a household's claim risk is not a function of its choice of deductible. That is, we assume households do not suffer from moral hazard. In particular, we assume there is neither ex ante moral hazard (deductible choice does not influence the frequency of claimable events) nor ex post moral hazard (deductible choice does not influence the decision to file a claim). The empirical evidence on moral hazard in auto insurance markets is mixed (Cohen \& Siegelman 2010), and we are not aware of any empirical evidence on moral hazard in home insurance markets. Because deductibles are small relative to the overall level of coverage, it seems reasonable to assume there is no ex ante moral hazard. However, because the damage from a claimable event may occasionally be less than the chosen deductible (at least for "high deductible" households), it may be less reasonable to assume there is no ex post moral hazard. As a check of the sensitivity of the association parameter estimates to our assumption on moral hazard, we re-estimate the model separately for "low deductible" and "high deductible" households. We define a household as "low deductible" if none of its deductibles is greater than $\$ 250$. Conversely, we define a household as "high deductible" if at least one of its deductibles is greater than $\$ 250$. Table A8 in the Appendix reports the association parameter estimates for low and high deductible households. ${ }^{16}$ They are largely consistent with each other and with the estimates for the tricoverage sample, suggesting that moral hazard is not an issue. ${ }^{17}$

\section{Signaling Value of Claims Experience}

In this section, we demonstrate the value of the information contained in the estimated variance-covariance matrix $\widehat{\Sigma}$ —and, by implication, the signaling value of the households'

\footnotetext{
${ }^{16}$ As before, the re-estimations use GLM estimates of the regression parameters assuming the random effects follow a lognormal distribution.

${ }^{17}$ Our test of moral hazard is also a test of adverse selection, as adverse selection also implies a correlation between deductible choice and claim frequency (Chiappori \& Salanié 2000). Accordingly, the results reported in Table A8 in the Appendix also suggest that our estimates are robust to the possibility of adverse selection.
} 
claims histories - by showing that conditioning on claims experience leads to material refinements of the predicted claim rates in the tricoverage sample. We also demonstrate the incremental value of utilizing the information on the cross-coverage correlation structure of unobserved heterogeneity $(\rho)$, as opposed to utilizing only the information on the withincoverage variance of unobserved heterogeneity $\left(\sigma^{2}\right)$, by showing that conditioning across lines of coverage (in addition to conditioning within lines of coverage) leads to material incremental refinements of the predicted claim rates.

Throughout this section and beyond, we distinguish among three types of predicted claim rates. The first are prior claim rates, $\widehat{\lambda}_{i t k} \equiv \exp \left(\mathbf{x}_{i t k}^{\prime} \widehat{\beta}_{k}\right)$. These are a priori predicted claim rates based on ex ante observables. The second are uniline posterior claim rates, $\widehat{\vartheta}_{i t k} \equiv$ $\widehat{\lambda}_{i t k} E^{U L}\left(\epsilon_{i k} \mid \mathbf{y}_{i k}\right)$ for each coverage $k=c, m, h$, where $\mathbf{y}_{i k} \equiv\left(y_{i 1 k}, \ldots, y_{i T_{i} k}\right)$ and $E^{U L}\left(\epsilon_{i k} \mid \mathbf{y}_{i k}\right)$ is calculated assuming $\epsilon_{i k} \stackrel{i i d}{\sim}$ lognormal with $E\left(\epsilon_{i k}\right)=1$ and $V\left(\epsilon_{i k}\right)=\widehat{\sigma}_{k}^{2}$. These are a posteriori predicted claim rates conditional on within-coverage ex post claims experience. The third are multiline posterior claim rates, $\widehat{\theta}_{i t k} \equiv \widehat{\lambda}_{i t k} E^{M L}\left(\epsilon_{i k} \mid \mathbf{y}_{i}\right)$, where $\mathbf{y}_{i} \equiv\left(\mathbf{y}_{i c}, \mathbf{y}_{i m}, \mathbf{y}_{i h}\right)$ and $E^{M L}\left(\epsilon_{i k} \mid \mathbf{y}_{i}\right)$ is calculated assuming $\epsilon_{i} \equiv\left[\begin{array}{lll}\epsilon_{i c} & \epsilon_{i m} & \epsilon_{i h}\end{array}\right]^{\prime} \stackrel{\text { iid }}{\sim}$ lognormal with $E\left(\epsilon_{i}\right)=\left[\begin{array}{lll}1 & 1 & 1\end{array}\right]^{\prime}$ and $V\left(\epsilon_{i}\right)=\widehat{\Sigma}$. These are a posteriori predicted claim rates conditional on ex post claims experience both within and across lines of coverages. The derivations of $E^{U L}\left(\epsilon_{i k} \mid \mathbf{y}_{i k}\right)$ and $E^{M L}\left(\epsilon_{i k} \mid \mathbf{y}_{i}\right)$ are set forth in the Appendix.

Our approach to updating the prior claim rates accords with standard Bayesian credibility theory. In actuarial parlance, credibility refers to statistical methods that combine individual and class estimates of the risk premium (i.e., the expected number of claims). Bayesian credibility refers to credibility approaches that utilize Bayes' theorem. The Bayesian credibility premium is the a priori predicted claim rate (the class estimate) multiplied by an a posteriori correction - also known as a bonus-malus coefficient - that reflects idiosyncratic claim risk (the individual estimate). In particular, $\widehat{\vartheta}_{i t k} \equiv \widehat{\lambda}_{i t k} E^{U L}\left(\epsilon_{i k} \mid \mathbf{y}_{i k}\right)$ corresponds to the textbook Bayesian credibility premium-where $E^{U L}\left(\epsilon_{i k} \mid \mathbf{y}_{i k}\right)$ is the bonus-malus coefficient-for the single-line Poisson-lognormal credibility model, and $\widehat{\theta}_{i t k} \equiv \widehat{\lambda}_{i t k} E^{M L}\left(\epsilon_{i k} \mid \mathbf{y}_{i}\right)$ is the extension to 
the multiple-line case. A noteworthy property of the Bayesian credibility approach is that it is balanced: $E\left(E^{U L}\left(\epsilon_{i k} \mid \mathbf{y}_{i k}\right)\right)=1$ and $\left.E\left(E^{M L}\left(\epsilon_{i k} \mid \mathbf{y}_{i}\right)\right)\right)=1$ for each coverage $k \in\{c, m, h\}$, so the bonus-malus corrections average to unity. For more on Bayesian credibility theory, see generally, e.g., Denuit et al. (2007, ch. 3).

To demonstrate that conditioning on claims experience leads to material refinements of the predicted claim rates, we compare the empirical distribution of the prior claim rates, $\widehat{\lambda}_{i t k}$, with that of the multiline posterior claim rates, $\widehat{\theta}_{i t k}$. Figure 1 plots, for each coverage $k$, the kernel density of $\eta_{i t k} \equiv\left(\widehat{\theta}_{i t k}-\widehat{\lambda}_{i t k}\right) / \widehat{\lambda}_{i t k}$. Further details are set forth in Table 2. For households with negative values of $\eta_{i t k}$, the mean value of $\eta_{i t k}$ is -7 percent in auto collision, -13 percent in auto comprehensive, and -14 percent in home. For a quarter of these households, $\eta_{i t k}$ is less than-9 percent in auto collision, -19 percent in auto comprehensive, and -20 percent in home. For a tenth, $\eta_{i t k}$ is less than -12 percent in auto collision and -24 percent in both auto comprehensive and home. The numbers are even more striking for households with positive values of $\eta_{i t k}$. For these households, the mean value of $\eta_{i t k}$ is +10 percent in auto collision, +23 percent in auto comprehensive, and +28 percent in home. For a quarter of these households, $\eta_{i t k}$ exceeds +14 percent in auto collision, +31 percent in auto comprehensive, and +37 percent in home. For a tenth, $\eta_{i t k}$ exceeds +23 percent in auto collision, +53 percent in auto comprehensive, and +65 percent in home. The numbers are similar for households with low, medium, and high prior claim rates, ${ }^{18}$ suggesting that the value of the information in $\widehat{\Sigma}$ is robust to differences in baseline claim risk.

To show the incremental value of conditioning across lines of coverage, we compare the empirical distribution of the multiline posterior claim rates, $\widehat{\theta}_{i t k}$, with that of the uniline posterior claim rates, $\widehat{\vartheta}_{i t k}$. Figure 2 plots, for each coverage $k$, the kernel density of $\zeta_{i t k} \equiv$ $\left(\widehat{\theta}_{i t k}-\widehat{\vartheta}_{i t k}\right) / \widehat{\vartheta}_{i t k}$. Further details are set forth in Table 3. For households with negative values of $\zeta_{i t k}$, the mean value of $\zeta_{i t k}$ is -3 percent in auto collision, -10 percent in auto

\footnotetext{
${ }^{18}$ A prior claim rate is "low" if it is in the bottom quartile and "high" if it is in the top quartile. It is "medium" otherwise. In the tricoverage sample, the respective low and high cutoffs are 0.078 and 0.127 in auto collision, 0.016 and 0.044 in auto comprehensive, and 0.054 and 0.096 in home.
} 
comprehensive, and -4 percent in home, and for a tenth $\zeta_{i t k}$ is less than -6 percent in auto collision, -17 percent in auto comprehensive, and -8 percent in home. Again, the numbers are more striking for households with positive values of $\eta_{i t k}$. For these households, the mean value of $\zeta_{i t k}$ is +7 percent in auto collision, +16 percent in auto comprehensive, and +9 percent in home, and for a tenth $\zeta_{i t k}$ exceeds +15 percent in auto collision, +36 percent in auto comprehensive, and +21 percent in home. As before, the numbers are similar for households with low, medium, and high prior claim rates, suggesting that the incremental value of the information in $\widehat{\rho}$ is robust to differences in baseline claim risk.

\section{Experience Rating And Deductible Choices}

The previous section demonstrates the signaling value of the households' claims experience. In this section, we investigate the extent to which the households' demand for insurance coverage, as captured by their deductible choices, would respond to experience rating. As noted above, this yields a lower bound on the potential for unpriced heterogeneity to distort their demand for insurance coverage, which in turn sheds light on the potential for experience rating to reduce market inefficiency due to unobserved heterogeneity in claim risk.

\section{A. Experience Rating}

In each coverage, the company uses the same basic procedure to generate a household's pricing menu of premium-deductible combinations. The company first determines a household's base price $\bar{p}$ according to a coverage-specific rating function, which takes into account the household's coverage-relevant characteristics and any applicable discounts. Using the base price, the company then generates the household's pricing menu $\mathcal{M}=\{(p(d), d): d \in \mathcal{D}\}$, which associates a premium $p(d)$ with each deductible $d$ in the coverage-specific set of deductible options $\mathcal{D}$, according to a coverage-specific multiplication rule, $p(d)=(g(d) \cdot \bar{p})+\delta$, where $g(\cdot)$ is a decreasing positive function and $\delta>0$. The multiplicative factors $\{g(d): d \in$ 
$\mathcal{D}\}$ are known as the deductible factors and $\delta$ is a small markup known as the expense fee. The deductible factors and the expense fee are coverage specific but household invariant.

We assume that the company's experience rating scheme is a simple bonus-malus system under which base prices are adjusted in proportion to changes in predicted claim risk. Let $\bar{p}_{i k}^{U L}$ denote household $i$ 's experience rated base price in coverage $k$ in the case of uniline experience rating (i.e., when premiums are experience rated only within coverages), and let $\bar{p}_{i k}^{M L}$ denote household $i$ 's experience rated base price in coverage $k$ in the case of multiline experience rating (i.e., when premiums are experience rated both within and across coverages). We assume that

$$
\bar{p}_{i k}^{U L}=\frac{\widehat{\vartheta}_{i k}}{\widehat{\lambda}_{i k}} \times \bar{p}_{i k} \quad \text { and } \quad \bar{p}_{i k}^{M L}=\frac{\widehat{\theta}_{i k}}{\widehat{\lambda}_{i k}} \times \bar{p}_{i k}
$$

where $\widehat{\lambda}_{i k}, \widehat{\vartheta}_{i k}$, and $\widehat{\theta}_{i k}$ denote household $i$ 's prior claim rate, uniline posterior claim rate, and multiline posterior claim rate, respectively, in coverage $k$. Recall that $\widehat{\vartheta}_{i k}=\widehat{\lambda}_{i k} E^{U L}\left(\epsilon_{i k} \mid \mathbf{y}_{i k}\right)$ and $\widehat{\theta}_{i k}=\widehat{\lambda}_{i k} E^{M L}\left(\epsilon_{i k} \mid \mathbf{y}_{i}\right)$. Hence,

$$
\bar{p}_{i k}^{U L}=E^{U L}\left(\epsilon_{i k} \mid \mathbf{y}_{i k}\right) \times \bar{p}_{i k} \quad \text { and } \quad \bar{p}_{i k}^{M L}=E^{M L}\left(\epsilon_{i k} \mid \mathbf{y}_{i}\right) \times \bar{p}_{i k},
$$

where $E^{U L}\left(\epsilon_{i k} \mid \mathbf{y}_{i k}\right)$ and $E^{M L}\left(\epsilon_{i k} \mid \mathbf{y}_{i}\right)$ are the bonus-malus coefficients in the single-line and multiple-line Poisson-lognormal credibility models, respectively (see, e.g., Denuit et al. 2007, ch. 3; Pinquet 2013). Table 4 summarizes the uniline and multiline bonus-malus coefficients. Note that the uniline and multiline experience rating schemes are balanced in that the bonusmalus coefficients average to unity in each coverage.

\section{B. Deductible Choices}

We proceed in two steps to investigate how households' deductible choices would respond to experience rating. First, we assume that households make deductible choices according 
to standard expected utility theory. Second, we investigate how deductible choices would change when we move from prior premiums to uniline premiums and multiline premiums. ${ }^{19}$

\section{A Model of Deductible Choice}

A household $i$ faces a menu of premium-deductible pairs $\left\{\left(p_{i}(d), d\right): d \in \mathcal{D}\right\}$. The household experiences at most one claim during the policy period, and it believes the probability of experiencing a claim is $\mu_{i}$. In the event of a claim, the loss exceeds the maximum deductible option and payment of the deductible is the only cost associated with the claim. Under these assumptions, the household's choice of deductible involves a choice among lotteries of the form $L_{i}(d) \equiv\left(-p_{i}(d), 1-\mu_{i} ;-p_{i}(d)-d, \mu_{i}\right)$.

Under the standard expected utility model, the utility of lottery $L_{i}(d)$ is given by

$$
U_{i}\left(L_{i}(d)\right)=\left(1-\mu_{i}\right) u_{i}\left(w_{i}-p_{i}(d)\right)+\mu_{i} u_{i}\left(w_{i}-p_{i}(d)-d\right)
$$

where $u_{i}(\cdot)$ is the household's Bernoulli utility function and $w_{i}$ is its wealth. We assume that household $i$ chooses a deductible $d \in \mathcal{D}$ to maximize $U_{i}\left(L_{i}(d)\right)$.

We assume that every household has the same utility function: $u_{i}(\cdot)=u(\cdot)$ for all $i .^{20}$ For $u(\cdot)$, we consider a second-order Taylor expansion. Also, because $u(\cdot)$ is unique only up to an affine transformation, we normalize the scale of utility by dividing by $u^{\prime}(\cdot)$. With this specification, equation (1) becomes

$$
U_{i}\left(L_{i}(d)\right)=-\left[p_{i}(d)+\mu_{i} d\right]-\frac{r}{2}\left[\left(1-\mu_{i}\right)\left(p_{i}(d)\right)^{2}+\mu_{i}\left(p_{i}(d)+d\right)^{2}\right]
$$

where $r=-u^{\prime \prime}(\cdot) / u^{\prime}(\cdot)$ is the coefficient of absolute risk aversion.

\footnotetext{
${ }^{19}$ In the Appendix, we also consider a generalization of the expected utility model that allows for probability distortions.

${ }^{20}$ Despite the simplistic assumption of homogeneous preferences, the model fits the data reasonably well. It correctly predicts 53 percent of the deductible choices in home, 37 percent in auto collision, and 27 percent in auto comprehensive.
} 
We calibrate the model with the estimate for $r$ reported by Barseghyan et al. (2013) [hereafter, BMOT]. BMOT estimate equation (2) using a cross section of 4,170 households in the tricoverage sample. They arrive at their estimation sample by imposing two restrictions. First, they restrict attention to households who first purchased their auto and home policies in the same year, in either 2005 or 2006. This is meant to avoid temporal issues, such as changes in household characteristics and in the economic environment. Second, they consider only the initial deductible choices of each household. This is meant to increase confidence that they are working with active choices; one might be concerned that some households renew their policies without actively reassessing their deductible choices. To account for observationally equivalent households choosing different deductibles, BMOT assume random utility with additively separable choice noise. Specifically, they assume that the utility from deductible $d \in \mathcal{D}$ is $\mathcal{U}(d) \equiv U\left(L_{i}(d)\right)+\varepsilon_{i, d}$, where $\varepsilon_{i, d}$ follows a type 1 extreme value distribution with scale parameter $\sigma$. In addition, they assume that $\mu_{i}=1-\exp \left(-\widehat{\vartheta}_{i}\right)$. Estimating the model by maximum likelihood, they report $\widehat{r}=0.0129$.

\section{Results}

We use the calibrated model to investigate the extent to which the households' deductible choices would change if premiums were experience rated. In particular, we examine how the distribution of model-predicted deductible choices changes when we counterfactually move from prior premiums (i.e., premiums generated using non-experience rated base prices, $\bar{p}_{i k}$ ) to uniline premiums and multiline premiums (i.e., premiums generated using uniline experienced rated base prices, $\bar{p}_{i k}^{U L}$, and multiline experienced rated base prices, $\bar{p}_{i k}^{M L}$ ). In each case, we assume that households believe their claim probability is $\mu_{i}=1-\exp \left(-\widehat{\theta}_{i}\right)$.

Table 5 presents the results. In addition to displaying the distributions, the table reports the percentage of policies in which the deductible choice changes when we move from prior premiums to uniline and multiline premiums, as well as the resulting (absolute) changes in coverage. The main takeaway is that the response of deductible choices to experience 
rating would be substantial. When we move from prior premiums to uniline premiums, the deductible choice changes in 5 percent of auto collision policies, 12 percent of auto comprehensive policies, and 14 percent of home policies, resulting in average changes in coverage of $\$ 247, \$ 164$, and $\$ 343$, respectively, among policies with a change, and of $\$ 13$, $\$ 20$, and $\$ 47$, respectively, among all policies. When we move from prior premiums to multiline premiums, the deductible choice changes in 7 percent of auto collision policies, 21 percent of auto comprehensive policies, and 15 percent of home policies, resulting in average changes in coverage of $\$ 247, \$ 178$, and $\$ 347$, respectively, among policies with a change and of $\$ 17, \$ 38$, and $\$ 51$, respectively, among all policies. ${ }^{21}$

\section{CONCLUSION}

We examine the cost of legal restrictions on experience rating in auto and home insurance. Using data on claims histories, we first estimate the variance-covariance matrix of unobserved heterogeneity in claim risk. We find, inter alia, that unobserved heterogeneity in claim risk is positively correlated across coverages. As noted above, this suggests that there is a latent, domain-general component to risk type. This finding adds to a growing body of evidence that riskiness is a trans-substantive characteristic of individuals (e.g., Barksy et al. 1997; Dohmen et al. 2011; Golden et al. 2016). It also complements existing research suggesting that risk aversion, though not completely stable across contexts (Barseghyan et al. 2011), also has latent, domain-general component (e.g., Einav et al. 2012; Barseghyan et al. 2016). ${ }^{22}$

Next, we show that conditioning on claims experience leads to material refinements of predicted claim rates, with the average downward revisions ranging from 7 percent to 14 percent and the average upward revisions ranging from 10 percent to 28 percent. Accordingly, for the average household - who has prior predicted claim rates of 10.7 percent in auto collision, 3.2 percent in auto comprehensive, and 7.9 percent in home-such conditioning

\footnotetext{
${ }^{21}$ The results for the model with probability distortions are reported in the Appendix. The main takeaway is the same.

${ }^{22}$ But see Barseghyan et al. (2018).
} 
implies posterior predicted claim rates of 10.0 percent, 2.8 percent, and 6.8 percent, respectively, in the case of downward revisions and of 11.8 percent, 3.9 percent, and 10.1 percent, respectively, in the case of upward revisions.

Lastly, we assess how insureds' demand for coverage would respond to experience rating assuming that households make deductible choices according to standard expected utility theory. We find that the demand response to experience rating would be large. Across the three coverages, we find that anywhere from 7 percent to 21 percent of households would change their deductible in response to multiline experience rating, resulting in average changes in coverage ranging from $\$ 178$ to $\$ 347$ among policies with a change.

The main takeaway from our analysis is that in the absence of experience rating there would be considerable unpriced heterogeneity in claim risk that in turn would lead to sizable distortions in households' demand for insurance coverage. In other words, our analysis suggests that the cost of legal restrictions on experience rating can be substantial. We believe this is important for policymakers to keep in mind. As noted above, regulation of experience rating is widespread in the United States. In auto and home insurance, New York is just one of many states that limit insurers' ability to engage in experience rating. ${ }^{23}$ For instance, California and Oklahoma prohibit increasing an insured's premium for auto collision insurance on the basis of an accident for which the insured was not at fault; ${ }^{24}$ Florida and Pennsylvania prohibit increasing an insured's premium for auto comprehensive insurance on the basis of an accident whether or not the insured was at fault; ${ }^{25}$ and Oklahoma and Texas prohibit increasing an insured's premium for home insurance on the basis of a weather related claim or the first non-weather related claim. ${ }^{26}$

\footnotetext{
${ }^{23}$ As noted above, New York forbids experience rating of premiums for auto comprehensive or home insurance coverage and also prohibits using auto comprehensive claims to experience rate premiums in any other line of insurance coverage. See N.Y. Ins. Law $\S 2334$ (2018); N.Y. Comp. Codes R. \& Regis. tit. 11, $\S \S 161.8$ \& 169.1 (2018).

${ }^{24}$ See Cal. Ins. Code $\S \S 1861.02-.025$ (2018); Cal. Code Regs. tit. 10, $\S \S 2632.12-13.1$ (2018); Okla. Stat. tit. $36, \S 941$ (2018).

${ }^{25}$ See Fla. Stat. ch. 626.9541(o)(10) (2018); 31 Pa. Code $\S 67.33(b)(3)$ (2018).

${ }^{26}$ See Okla. Stat. tit. 36, § 3691.1 (2018); Okla. Admin. Code § 365:15-7-26 (2018); 28 Tex. Admin. Code $\S 21.1004$ (2018).
} 
Of course, in order to determine whether any particular legal restriction on experience rating is worth the cost, a policymaker would need to conduct a full-fledged welfare analysis (assuming she takes a welfarist approach to policymaking). Although such an analysis is beyond the scope of this paper, we can sketch out what it would entail. First and foremost, one would have to specify a social welfare function, which presumably would take into account the utility of both insureds and insurers. In addition, one would have to model and predict not only the behavior of insureds on the intensive margin (as we do), but also the behavior of insureds on the extensive margin and the behavior of insurers. This is because legal restrictions on experience rating can have long-term market effects beyond the shortterm demand effects that we consider. For instance, they can lead to risk-based adverse selection and higher insurance costs. Indeed, several studies of the auto insurance market find that insurance costs are higher when rate regulation generates cross-subsidies from lowto high-risk insureds (e.g., Weiss et al. 2010; Derrig \& Tennyson 2011). Needless to say, one would have to make difficult normative judgments in specifying the social welfare function. Moreover, the positive analysis would be sensitive to the assumptions that one makes about behavior. This is a topic that we hope to explore in future research.

\section{REFERENCES}

Abraham, Kenneth S. (1985) "Efficiency and Fairness in Insurance Risk Classification," 71 Virginia Law Review 403-451.

Abraham, Kenneth S., \& Pierre-André Chiappori (2015) "Classification Risk and its Regulation," in Daniel Schwarcz \& Peter Siegelman, eds., Research Handbook on the Economics of Insurance Law, Chap. 9. Cheltenham: Edward Elgar.

Angers, Jean-Franois, Denise Desjardins, Georges Dionne, \& Franois Guertin (2006) "Vehicle and Fleet Random Effects in a Model of Insurance Rating for Fleets of Vehicles," 36 ASTIN Bulletin 25-77.

Antonio, Katrien, Montserrat Guilln, \& Ana-Maria Prez Marn (2011) "Multidimensional Credibility: A Bayesian Analysis of Policholders Holding Multiple Contracts," Working Paper, University of Amsterdam. 
Antonio, Katrien, \& Emiliano A. Valdez (2012) "Statistical Concepts of A Priori and A Posteriori Risk Classification in Insurance," 96 AstA Advances in Statistical Analysis $187-224$.

Avraham, Ronen, Kyle D. Logue, \& Daniel Schwarcz (2014) "Understanding Insurance AntiDiscrimination Laws," 87 Southern California Law Review 195-274.

Barksy, Robert B., F. Thomas Juster, Miles S. Kimball, \& Matthew D. Shapiro (1997) "Preference Parameters and Behavioral Heterogeneity: An Experimental Approach in the Health and Retirement Study," 112 Quarterly Journal of Economics 537-579.

Barseghyan, Levon, Francesca Molinari, Ted O'Donoghue, \& Joshua C. Teitelbaum (2013) "The Nature of Risk Preferences: Evidence from Insurance Choices," 103 American Economic Review 2499-2529.

Barseghyan, Levon, Francesca Molinari, \& Joshua C. Teitelbaum (2016) "Inference under Stability of Risk Preferences," 7 Quantitative Economics 367-409.

Barseghyan, Levon, Jeffrey Prince, \& Joshua C. Teitelbaum (2011) "Are Risk Preferences Stable Across Contexts? Evidence from Insurance Data," 101 American Economic Review 591-631.

Barseghyan, Levon, Joshua C. Teitelbaum, \& Lin Xu (2018) "Different Contexts, Different Risk Preferences?," Working Paper, Cornell University.

Bhlmann, Hans, \& Alois Gisler (2005) A Course in Credibility Theory and its Applications. Berlin: Springer.

Buchmueller, Thomas, \& John DiNardo (2002) "Did Community Rating Induce an Adverse Selection Death Spiral? Evidence from New York, Pennsylvania, and Connecticut," 92 American Economic Review 280-294.

Bundorf, M. Kate, Jonathan Levin, \& Neale Mahoney (2012) "Pricing and Welfare in Health Plan Choice," 102 American Economic Review 3214-3248.

Bundorf, M. Kate, \& Kosali I. Simon (2006) "The Effects of Rate Regulation on Demand for Supplemental Health Insurance," 96 American Economic Review: Papers and Proceedings $67-71$.

Chiappori, Pierre-André, \& Bernard Salanié (2000) "Testing for Asymmetric Information in Insurance Markets," 108 Journal of Political Economy 56-78. 
Cohen, Alma, \& Peter Siegelman (2010) "Testing for Adverse Selection in Insurance Markets," 77 Journal of Risk and Insurance 39-84.

Crocker, Keith J., \& Arthur Snow (1986) "The Efficiency Effects of Categorical Discrimination in the Insurance Industry," 94 Journal of Political Economy 321-344.

(2011) "Multidimensional Screening in Insurance Markets with Adverse Selection," 78 Journal of Risk and Insurance 287-307.

Denuit, Michel, Xavier Marchal, Sandra Pitrebois, \& Jean-Franois Walhin (2007) Actuarial Modelling of Claim Counts: Risk Classification, Credibility and Bonus-Malus Systems. Chichester: John Wiley.

Derrig, Richard A., \& Sharon Tennyson (2011) "The Impact of Rate Regulation on Claims: Evidence from Massachusetts Automobile Insurance," 14 Risk Management and Insurance Review 173-199.

Desjardins, Denise, Georges Dionne, \& Jean Pinquet (2001) "Experience Rating Schemes for Fleets of Vehicles," 31 ASTIN Bulletin 81-105.

Dionne, Georges, \& Casey Rothschild (2014) "Economic Effects of Risk Classification Bans," 39 Geneva Risk and Insurance Review 184-221.

Dohmen, Thomas, Armin Falk, David Huffman, Uwe Sunde, Jrgen Schupp, \& Gert G. Wagner (2011) "Individual Risk Attitudes: Measurement, Determinants, and Behavioral Consequences," 9 Journal of the European Economic Association 522-550.

Einav, Liran, Amy Finkelstein, \& Mark R. Cullen (2010) "Estimating Welfare in Insurance Markets Using Variation in Prices," 125 Quarterly Journal of Economics 877-921.

Einav, Liran, Amy Finkelstein, Iuliana Pascu, \& Mark R. Cullen (2012) "How General are Risk Preferences? Choice under Uncertainty in Different Domains," 102 American Economic Review 2606-2638.

Englund, Martin, Montserrat Guilln, Jim Gustafsson, Jens Perch Nielsen, \& Fredrik Thuring (2009) "Multidimensional Credibility with Time Effects: An Application to Commerical Business Lines," 76 Journal of Risk and Insurance 443-453.

Englund, Martin, Montserrat Guilln, Jim Gustafsson, Lars Hougaard Nielsen, \& Jens Perch Nielsen (2008) "Multivariate Latent Risk: A Credibility Approach," 38 ASTIN Bulletin $137-146$. 
Finkelstein, Amy, James Poterba, \& Casey Rothschild (2009) "Redistribution by Insurance Market Regulation: Analyzing a Ban on Gender-Based Retirement Annuities," 91 Journal of Financial Economics 38-58.

Frees, Edward J. (2003) "Multivariate Credibility for Aggregate Loss Models," 7 North American Actuarial Journal 13-37.

Frees, Edward J., Glenn Meyers, \& A. David Cummings (2010) "Dependent Multi-Peril Ratemaking Models," 40 ASTIN Bulletin 699-726.

Geruso, Michael (2017) "Demand Heterogeneity in Insurance Markets: Implications for Equity and Efficiency," 8 Quantitative Economics 929-975.

Golden, Linda L., Patrick L. Brockett, Jing Ai, \& Bruce Kellison (2016) "Empirical Evidence on the Use of Credit Scoring for Predicting Insurance Losses with Psycho-social and Biochemical Explanations," 20 North American Actuarial Journal 233-251.

Gouriroux, Christian, Alain Monfort, \& Alain Trognon (1984a) "Psuedo Maximum Likelihood Methods," 52 Econometrica 681-700.

(1984b) "Psuedo Maximum Likelihood Methods: Applications to Poisson Models," 52 Econometrica 701-720.

Gul, Faruk (1991) "A Theory of Disappointment Aversion," 59 Econometrica 667-686.

Hoy, Michael (1982) "Categorizing Risks in the Insurance Industry," 97 Quarterly Journal of Economics 321-336.

_ (2006) "Risk Classification and Social Welfare," 31 Geneva Papers on Risk and Insurance: Issues and Practice 245-269.

Jewell, William S. (1974) "Exact Multidimensional Credibility," 74 Bulletin of Swiss Association of Actuaries 193-214.

Kőszegi, Botond, \& Matthew Rabin (2006) "A Model of Reference-Dependent Preferences," 121 Quarterly Journal of Economics 1133-1166.

_ (2007) "Reference-Dependent Risk Attitudes," 97 American Economic Review 10471073.

Lemaire, Jean (1995) Bonus-Malus Systems in Automobile Insurance. Boston: Kluwer. 
Liang, Kung-Yee, \& Scott L. Zeger (1986) "Longitudinal Data Analysis Using Generalized Linear Models," 73 Biometrika 13-22.

Meier, Kenneth J. (1991) "The Politics of Insurance Regulation," 58 Journal of Risk and Insurance 700-713.

Morris, Darcy Steeg (2012) "A Semiparametric Approach for Multivariate Longitudinal Count Data," in Methods for Multivariate Longitudinal Count and Duration Models with Applications in Economics, Chap. 2. Ph.D. Dissertation, Cornell University.

Pinquet, Jean (1998) "Designing Optimal Bonus-Malus Systems from Different Types of Claims," 28 ASTIN Bulletin 205-220.

- (2000) "Experience Rating Through Heterogeneous Models," in Georges Dionne, ed., Handbook of Insurance, Chap. 14. Boston: Kluwer.

(2013) "Experience Rating in Non-Life Insurance," in Georges Dionne, ed., Handbook of Insurance, Second Edition, Chap. 17. New York: Springer.

Prentice, Ross L. (1988) "Correlated Binary Regression with Covariates Specific to Each Binary Observation," 44 Biometrics 1033-1048.

Quiggin, John (1982) "A Theory of Anticipated Utility," 3 Journal of Economic Behavior and Organization 323-343.

Rothschild, Casey (2011) "The Efficiency of Categorical Discrimination in Insurance Markets," 78 Journal of Risk and Insurance 267-285.

Salani, Bernard (1997) The Economics of Contracts: A Primer. Cambridge, MA: MIT Press.

Simon, Kosali Ilayperuma (2005) "Adverse Selection in Health Insurance Markets? Evidence from State Small-Group Health Insurance Reforms," 89 Journal of Public Economics 18651877.

Thiery, Yves, \& Caroline Van Schoubroeck (2006) "Fairness and Equality and Insurance Classification," 31 Geneva Papers on Risk and Insurance: Issues and Practice 190-211.

Thomas, R. Guy (2007) "Some Novel Perspectives on Risk Classification," 32 Geneva Papers on Risk and Insurance: Issues and Practice 105-132.

_ (2008) "Loss Coverage as a Public Policy Objective for Risk Classification Schemes," 75 Journal of Risk and Insurance 997-1018. 
Thuring, F., J.P. Nielsen, M. Guilln, \& C. Bolanc (2012) "Selecting Prospects for Crossselling Financial Products Using Multivariate Credibility," 39 Expert Systems with Applications 8809-8816.

Thuring, Fredrik (2011) "A Credibility Method for Profitable Cross-selling of Insurance Products," 6 Annals of Actuarial Science 65-75.

Tversky, Amos, \& Daniel Kahneman (1992) "Advances in Prospect Theory: Cumulative Representation of Uncertainty," 5 Journal of Risk and Uncertainty 297-323.

Vuong, Quang H. (1989) "Likelihood Ratio Tests for Model Selection and Non-Nested Hypotheses," 57 Econometrica 307-333.

Weiss, Mary A., Sharon Tennyson, \& Laureen Regan (2010) "The Effects of Regulated Premium Subsidies on Insurance Costs: An Empirical Analysis of Automobile Insurance," 77 Journal of Risk and Insurance 597-624.

Wooldridge, Jeffrey M. (2002) Econometric Analysis of Cross Section and Panel Data. Cambridge, MA: MIT Press.

Zeger, Scott L., \& Kung-Yee Liang (1986) "Longitudinal Data Analysis for Discrete and Continuous Outcomes," 42 Biometrics 121-130.

Zeger, Scott L., Kung-Yee Liang, \& Paul S. Albert (1988) "Models for Longitudinal Data: A Generalized Estimating Equation Approach," 44 Biometrics 1049-1060.

Ziegler, Andreas (2011) Generalized Estimating Equations. New York: Springer.

\section{Appendix}

\section{A. Descriptive Statistics}

Table A1 provides descriptive statistics for the tricoverage sample. Tables A2, A3, and A4 summarize the claims, premiums, and deductibles in the tricoverage sample.

\section{B. Estimation Approach}

Let $y_{i t k}$ denote the number of claims for household $i$ in year $t$ under coverage $k$, where $i=1, \ldots, N, t=1, \ldots, T_{i}$, and $k \in\{c, m, h\}$. Similarly, let $\mathbf{x}_{i t k}$ denote a vector of observables (plus a constant) for household $i$ in year $t$ under coverage $k$. Let $\lambda_{i t k}$ denote household $i$ 's 
baseline claim rate in year $t$ under coverage $k$, and let $\epsilon_{i k}$ denote a time-constant random effect for household $i$ under coverage $k$. Also, let $\mathbf{y}_{i k} \equiv\left(y_{i 1 k}, \ldots, y_{i T_{i} k}\right)$ and $\mathbf{y}_{i} \equiv\left(\mathbf{y}_{i c}, \mathbf{y}_{i m}, \mathbf{y}_{i h}\right)$, and let $\boldsymbol{\lambda}_{i k} \equiv\left(\lambda_{i 1 k}, \ldots, \lambda_{i T_{i} k}\right)$ and $\boldsymbol{\lambda}_{i} \equiv\left(\boldsymbol{\lambda}_{i c}, \boldsymbol{\lambda}_{i m}, \boldsymbol{\lambda}_{i h}\right)$.

The first two marginal moments for the class of models used in this research-longitudinal multivariate count models with multiplicative correlated random effects - are

$$
E\left(y_{i t k} \mid \mathbf{x}_{i t k}\right)=\exp \left(\mathbf{x}_{i t k}^{\prime} \boldsymbol{\beta}_{k}\right)=\lambda_{i t k}
$$

and

$$
\mathbf{V}_{i} \equiv \operatorname{Var}\left(\mathbf{y}_{i} \mid \mathbf{x}_{i}\right)=\operatorname{diag}\left(\boldsymbol{\lambda}_{i}^{\prime}\right)+\boldsymbol{\Sigma} \otimes \mathbf{1}_{T_{i}} \mathbf{1}_{T_{i}}^{\prime} \circ \boldsymbol{\lambda}_{i} \boldsymbol{\lambda}_{i}^{\prime}
$$

where $\circ$ is element-wise multiplication, $\otimes$ is the Kronecker product, and $\mathbf{1}_{T_{i}}$ is a $T_{i}$-dimensional vector of ones.

The moment-based approach for fitting this model relies on the moment conditions implied by the marginal mean and variance along with the basic assumptions for multiplicative correlated random effects models. The estimator $(\widehat{\boldsymbol{\beta}}, \widehat{\boldsymbol{\Sigma}})$ for $\boldsymbol{\beta} \equiv\left[\boldsymbol{\beta}_{c} \boldsymbol{\beta}_{m} \boldsymbol{\beta}_{h}\right]^{\prime}$ and $\boldsymbol{\Sigma}$ is defined as the solution to

$$
\sum_{i}\left(\begin{array}{cc}
\mathbf{D}_{i}^{\prime} & 0 \\
0 & \mathbf{E}_{i}^{\prime}
\end{array}\right)\left(\begin{array}{cc}
\mathbf{V}_{i} & 0 \\
0 & \mathbf{I}
\end{array}\right)^{-1}\left(\begin{array}{c}
\mathbf{y}_{i}-\boldsymbol{\lambda}_{i} \\
\mathbf{R}_{i}^{*}-\mathbf{V}_{i}^{*}
\end{array}\right)=0
$$

where

$$
\mathbf{D}_{i} \equiv \frac{\partial \boldsymbol{\lambda}_{i}}{\partial \boldsymbol{\beta}}=\operatorname{diag}\left[\mathbf{x}_{i c}^{\prime} \boldsymbol{\lambda}_{i c} \mathbf{x}_{i m}^{\prime} \boldsymbol{\lambda}_{i m} \mathbf{x}_{i h}^{\prime} \boldsymbol{\lambda}_{i h}\right]^{\prime}
$$

$\mathbf{V}_{i}$ is the model based variance-covariance matrix as defined above,

$$
\mathbf{E}_{i} \equiv \frac{\partial \mathbf{V}_{i}^{*}}{\partial \boldsymbol{\Sigma}^{*}}=\operatorname{diag}\left[\left(\boldsymbol{\lambda}_{i c} \boldsymbol{\lambda}_{i c}^{\prime}\right)^{*}\left(\boldsymbol{\lambda}_{i m} \boldsymbol{\lambda}_{i m}^{\prime}\right)^{*}\left(\boldsymbol{\lambda}_{i h} \boldsymbol{\lambda}_{i h}^{\prime}\right)^{*}\left(\boldsymbol{\lambda}_{i c} \boldsymbol{\lambda}_{i m}^{\prime}\right)^{*}\left(\boldsymbol{\lambda}_{i c} \boldsymbol{\lambda}_{i h}^{\prime}\right)^{*}\left(\boldsymbol{\lambda}_{i m} \boldsymbol{\lambda}_{i h}^{\prime}\right)^{*}\right]
$$

I is the identity matrix, and $\mathbf{R}_{i}$ is the cross product of residuals $r_{i t k} \equiv y_{i t k}-\lambda_{i t k}$. Also, let * indicate a half-vectorization operator, such that $\mathbf{R}_{i}^{*}, \mathbf{V}_{i}^{*}$, and $\boldsymbol{\Sigma}^{*}$ are the vectors of the upper triangular elements of the matrices $\mathbf{R}_{i}, \mathbf{V}_{i}$, and $\boldsymbol{\Sigma}$, respectively. The roots of the set of estimating equations are obtained via an iterative procedure, updated at each iteration with the consistent estimator of $\boldsymbol{\beta}$ given $\widehat{\boldsymbol{\Sigma}}$ and the consistent estimator of $\boldsymbol{\Sigma}$ given $\widehat{\boldsymbol{\beta}}$, until convergence. See Morris (2012) for more details on the estimation algorithm and asymptotic results for joint inference. 


\section{Regression Estimates}

Tables A5 (auto) and A6 (home) report the estimates of the regression parameters, $\beta$. Although $\beta$ is not the object of principal interest, the estimates reveal several noteworthy facts. First, auto claim rates (collision and comprehensive) are negatively related to insurance score (which is based on information contained in credit reports) but positively related to the age and number of vehicles. However, they are not correlated with vehicle safety features (passive restraint, anti-theft, and anti-lock brakes). Second, collision claim rates are negatively related to the age of the primary driver and are higher for households in which the primary driver is female. Conversely, comprehensive claim rates are positively related to the age of the primary driver and are lower for households in which the primary driver is female. Third, collision claim rates are higher for households with three or more drivers. Finally, home claim rates are negatively related to insurance score but positively related to the age and insured value of the home. In addition, they are higher for homes that are used for farming or business and for homes that are not the owner's primary residence. Home claim rates, however, are not correlated with home safety features (masonry construction, distance to fire hydrant, and alarm or other protection).

\section{Derivations of $E^{U L}\left(\epsilon_{i k} \mid \mathbf{y}_{i k}\right)$ and $E^{M L}\left(\epsilon_{i k} \mid \mathbf{y}_{i}\right)$}

Let $y_{i t k}$ denote the number of claims for household $i$ in year $t$ under coverage $k$, where $i=1, \ldots, N, t=1, \ldots, T_{i}$, and $k \in\{c, m, h\}$. Similarly, let $\mathbf{x}_{i t k}$ denote a vector of observables (plus a constant) for household $i$ in year $t$ under coverage $k$. Let $\lambda_{i t k}$ denote household $i$ 's baseline claim rate in year $t$ under coverage $k$, and let $\epsilon_{i k}$ denote a time-constant random effect for household $i$ under coverage $k$. Also, let $\mathbf{y}_{i k} \equiv\left(y_{i 1 k}, \ldots, y_{i T_{i} k}\right)$ and $\mathbf{y}_{i} \equiv\left(\mathbf{y}_{i c}, \mathbf{y}_{i m}, \mathbf{y}_{i h}\right)$.

1. Derivation of $E^{M L}\left(\epsilon_{i k} \mid \mathbf{y}_{i}\right)$

We assume

$$
y_{i t k} \mid \mathbf{x}_{i t k} \sim \text { Poisson }\left(\lambda_{i t k} \epsilon_{i k}\right)
$$

where

$$
\lambda_{i t k}=\exp \left(\mathbf{x}_{i t k}^{\prime} \beta_{k}\right)
$$


and $\epsilon_{i} \equiv\left[\epsilon_{i c} \epsilon_{i m} \epsilon_{i h}\right]^{\prime} \stackrel{i i d}{\sim}$ lognormal with $E\left(\epsilon_{i}\right)=\left[\begin{array}{lll}1 & 1 & 1\end{array}\right]^{\prime}$ and $V\left(\epsilon_{i}\right)=\Sigma$. This leads to the following probability distribution functions:

$$
\begin{aligned}
f\left(\mathbf{y}_{i} \mid \epsilon_{i}\right) & =\prod_{k} \prod_{t} \text { Poisson }\left(\lambda_{i t k} \epsilon_{i k}\right) \\
& =\prod_{k} \prod_{t} \frac{\left(\epsilon_{i k} \lambda_{i t k}\right)^{y_{i t k}}}{y_{i t k} !} e^{-\epsilon_{i k} \lambda_{i t k}} \\
& =\left(\prod_{k} \sum_{i k}^{\sum_{i k} y_{i t k}} e^{-\epsilon_{i k} \sum_{t} \lambda_{i t k}}\right)\left(\prod_{k} \prod_{t} \frac{\lambda_{i t k}^{y_{i t k}}}{y_{i t k} !}\right), \\
f\left(\tilde{\epsilon}_{i}\right) & =\operatorname{Normal}(\tilde{\mu}, \tilde{\boldsymbol{\Sigma}}) \\
& =\frac{1}{(2 \pi)^{3 / 2}}|\tilde{\boldsymbol{\Sigma}}|^{-1 / 2} e^{-\frac{1}{2}\left(\tilde{\epsilon}_{i}-\tilde{\mu}\right)^{\prime} \tilde{\boldsymbol{\Sigma}}^{-1}\left(\tilde{\epsilon}_{i}-\tilde{\mu}\right)},
\end{aligned}
$$

where $\tilde{\epsilon}_{i} \equiv \ln \left(\epsilon_{i}\right), \tilde{\mu} \equiv-\frac{\operatorname{diag}(\tilde{\boldsymbol{\Sigma}})}{2}$, and $\tilde{\boldsymbol{\Sigma}} \equiv \ln (\boldsymbol{\Sigma}+1)$, and

$$
\begin{aligned}
f\left(\mathbf{y}_{i}\right) & =\int_{\tilde{\epsilon}_{i c}} \int_{\tilde{\epsilon}_{i m}} \int_{\tilde{\epsilon}_{i h}} f\left(\mathbf{y}_{i} \mid \tilde{\epsilon}_{i}\right) f\left(\tilde{\epsilon}_{i}\right) d \tilde{\epsilon}_{i h} d \tilde{\epsilon}_{i m} d \tilde{\epsilon}_{i c} \\
& =\int_{\tilde{\epsilon}_{i c}} \int_{\tilde{\epsilon}_{i m}} \int_{\tilde{\epsilon}_{i h}} \prod_{k} \prod_{t} \operatorname{Poisson}\left(\lambda_{i t k} e^{\tilde{\epsilon}_{i k}}\right) M V N(\tilde{\mu}, \tilde{\Sigma}) d \tilde{\epsilon}_{i h} d \tilde{\epsilon}_{i m} d \tilde{\epsilon}_{i c} \\
& =\frac{1}{(2 \pi)^{3 / 2}}|\tilde{\Sigma}|^{-1 / 2}\left(\prod_{k} \prod_{t} \frac{\lambda_{i t k}^{y_{i t k}}}{y_{i t k} !}\right) \int_{\tilde{\epsilon}_{i c}} \int_{\tilde{\epsilon}_{i m}} \int_{\tilde{\epsilon}_{i h}} g^{M L}\left(\tilde{\epsilon}_{i}\right) d \tilde{\epsilon}_{i h} d \tilde{\epsilon}_{i m} d \tilde{\epsilon}_{i c}
\end{aligned}
$$

where $g^{M L}\left(\tilde{\epsilon}_{i}\right) \equiv\left(\prod_{k} e^{\tilde{\epsilon}_{i k} \sum_{t} y_{i t k}} e^{-e^{\tilde{\epsilon}_{i k}} \sum_{t} \lambda_{i t k}}\right) e^{-\frac{1}{2}\left(\tilde{\epsilon}_{i}-\tilde{\mu}\right)^{\prime} \tilde{\boldsymbol{\Sigma}}^{-1}\left(\tilde{\epsilon}_{i}-\tilde{\mu}\right)}$. Taken together, the posterior distribution is defined as

$$
\begin{aligned}
f\left(\tilde{\epsilon}_{i} \mid \mathbf{y}_{i}\right) & =\frac{f\left(\mathbf{y}_{i} \mid \tilde{\epsilon}_{i}\right) f\left(\tilde{\epsilon}_{i}\right)}{f\left(\mathbf{y}_{i}\right)} \\
& =\frac{g^{M L}\left(\tilde{\epsilon}_{i}\right)}{\int_{\tilde{\epsilon}_{i c}} \int_{\tilde{\epsilon}_{i m}} \int_{\tilde{\epsilon}_{i h}} g^{M L}\left(\tilde{\epsilon}_{i}\right) d \tilde{\epsilon}_{i h} d \tilde{\epsilon}_{i m} d \tilde{\epsilon}_{i c}}
\end{aligned}
$$

and the expectation is

$$
E^{M L}\left(\epsilon_{i} \mid \mathbf{y}_{i}\right)=\int_{\tilde{\epsilon}_{i c}} \int_{\tilde{\epsilon}_{i m}} \int_{\tilde{\epsilon}_{i h}}\left[\begin{array}{c}
e^{\tilde{\epsilon}_{i c}} \\
e^{\tilde{\epsilon}_{i m}} \\
e^{\tilde{\epsilon}_{i h}}
\end{array}\right] f\left(\tilde{\epsilon}_{i} \mid \mathbf{y}_{i}\right) d \tilde{\epsilon}_{i h} d \tilde{\epsilon}_{i m} d \tilde{\epsilon}_{i c}
$$


2. Derivation of $E^{U L}\left(\epsilon_{i k} \mid \mathbf{y}_{i k}\right)$

The univariate expectation, $E^{U L}\left(\epsilon_{i k} \mid \mathbf{y}_{i k}\right)$, is a special case of the multivariate expectation, $E^{M L}\left(\epsilon_{i} \mid \mathbf{y}_{i}\right)$. Replacing the conditional and marginal distribution functions with their univariate counterparts, the univariate posterior distribution is

$$
\begin{aligned}
f\left(\tilde{\epsilon}_{i k} \mid \mathbf{y}_{i k}\right) & =\frac{f\left(\mathbf{y}_{i k} \mid \tilde{\epsilon}_{i k}\right) f\left(\tilde{\epsilon}_{i k}\right)}{f\left(\mathbf{y}_{i k}\right)} \\
& =\frac{g^{U L}\left(\tilde{\epsilon}_{i k}\right)}{\int_{\tilde{\epsilon}_{i k}} g^{U L}\left(\tilde{\epsilon}_{i}\right) d \tilde{\epsilon}_{i k}}
\end{aligned}
$$

where $g^{U L}\left(\tilde{\epsilon}_{i k}\right) \equiv\left(e^{\tilde{\epsilon}_{i k} \sum_{t} y_{i t k}} e^{-e^{\tilde{\epsilon}_{i k}} \sum_{t} \lambda_{i t k}}\right) e^{-\frac{1}{2 \tilde{\sigma}_{k}^{2}}\left(\tilde{\epsilon}_{i k}-\tilde{\mu}_{k}\right)^{\prime}\left(\tilde{\epsilon}_{i k}-\tilde{\mu}_{k}\right)}$ and $k \in\{c, m, h\}$, and the univariate expectation is

$$
E^{U L}\left(\epsilon_{i k} \mid \mathbf{y}_{i k}\right)=\int_{\tilde{\epsilon}_{i k}} e^{\tilde{\epsilon}_{i k}} f\left(\tilde{\epsilon}_{i k} \mid \mathbf{y}_{i k}\right) d \tilde{\epsilon}_{i k}
$$

for $k \in\{c, m, h\}$.

\section{E. Robutness Checks}

Table A7 reports the association parameter estimates for alternative samples A and B. Alternative sample A comprises a balanced panel of households who purchased all three coverages (auto collision, auto comprehensive, and home). Alternative sample B comprises an unbalanced panel of households who purchased both auto coverages (collision and comprehensive).

Table A8 reports the association parameter estimates for low and high deductible households. A low deductible household is a household with no deductible greater than $\$ 250$. A high deductible household is a household with at least one deductible greater than $\$ 250$.

\section{F. Deductible Choices: Probability Distortion Model}

In the main text, we investigate how households' deductible choices would respond to experience rating under the assumption that households make deductible choice according to standard expected utility theory. Here, we investigate how households' deductible choices would respond to experience rating under the assumption that households make deductible choice according to a generalization of the expected utility model that allows for probability distortions. We refer to this model as the probability distortion model.

As before, a household $i$ faces a menu of premium-deductible pairs $\left\{\left(p_{i}(d), d\right): d \in \mathcal{D}\right\}$. The household experiences at most one claim during the policy period, and it believes the probability of experiencing a claim is $\mu_{i}$. In the event of a claim, the loss exceeds the 
maximum deductible option and payment of the deductible is the only cost associated with the claim. Under these assumptions, the household's choice of deductible involves a choice among lotteries of the form $L_{i}(d) \equiv\left(-p_{i}(d), 1-\mu_{i} ;-p_{i}(d)-d, \mu_{i}\right)$.

Under the probability distortion model, the utility of lottery $L_{i}(d)$ is given by

$$
U_{i}\left(L_{i}(d)\right)=\left(1-\Omega_{i}\left(\mu_{i}\right)\right) u_{i}\left(w_{i}-p_{i}(d)\right)+\Omega_{i}\left(\mu_{i}\right) u_{i}\left(w_{i}-p_{i}(d)-d\right),
$$

where $u_{i}(\cdot)$ is the household's Bernoulli utility function, $w_{i}$ is its wealth, and $\Omega_{i}(\cdot)$ is its probability distortion function. Given our setting, this model is quite general in that it includes several others as special cases, including models of subjective beliefs, rank-dependent probability weighting (Quiggin 1982; Tversky \& Kahneman 1992), loss aversion (Köszegi \& Rabin 2006, 2007), and disappointment aversion (Gul 1991). For further details about the probability distortion model, see Barseghyan et al. (2013) [hereafter, BMOT].

We assume that every household has the same utility and probability distortion function: $u_{i}(\cdot)=u(\cdot)$ and $\Omega_{i}(\cdot)=\Omega(\cdot)$ for all $i{ }^{27}$ For $u(\cdot)$, we consider a second-order Taylor expansion. Also, because $u(\cdot)$ is unique only up to an affine transformation, we normalize the scale of utility by dividing by $u^{\prime}(\cdot)$. With this specification, equation (A1) becomes

$$
U_{i}\left(L_{i}(d)\right)=-\left[p_{i}(d)+\Omega\left(\mu_{i}\right) d\right]-\frac{r}{2}\left[\left(1-\Omega\left(\mu_{i}\right)\right)\left(p_{i}(d)\right)^{2}+\Omega\left(\mu_{i}\right)\left(p_{i}(d)+d\right)^{2}\right],
$$

where $r=-u^{\prime \prime}(\cdot) / u^{\prime}(\cdot)$ is the coefficient of absolute risk aversion.

We calibrate the model with the estimates for $r$ and $\Omega(\cdot)$ reported by BMOT. They estimate equation (A2) using a cross section of 4,170 households in the tricoverage sample. They arrive at their estimation sample by imposing two restrictions. First, they restrict attention to households who first purchased their auto and home policies in the same year, in either 2005 or 2006. This is meant to avoid temporal issues, such as changes in household characteristics and in the economic environment. Second, they consider only the initial deductible choices of each household. This is meant to increase confidence that they are working with active choices; one might be concerned that some households renew their policies without actively reassessing their deductible choices. To account for observationally equivalent households choosing different deductibles, BMOT assume random utility with additively separable choice noise. Specifically, they assume that the utility from deductible $d \in \mathcal{D}$ is $\mathcal{U}(d) \equiv U\left(L_{i}(d)\right)+\varepsilon_{i, d}$, where $\varepsilon_{i, d}$ follows a type 1 extreme value distribution with scale parameter $\sigma$. In addition, they assume that $\Omega\left(\mu_{i}\right)=\exp \left(\delta_{0}+\delta_{1} \mu_{i}+\delta_{2} \mu_{i}^{2}\right)$ and

\footnotetext{
${ }^{27}$ Despite the simplistic assumption of homogeneous preferences, the model fits the data reasonably well. It correctly predicts 62 percent of deductible choices in home, 42 percent in auto collision, and 34 percent in auto comprehensive.
} 
$\mu_{i}=1-\exp \left(-\widehat{\vartheta}_{i}\right)$. Estimating the model by maximum likelihood, they report $\widehat{r}=0.00064$ and $\widehat{\Omega}\left(\mu_{i}\right)=\exp \left(-2.71+12.03 \mu_{i}-35.15 \mu_{i}^{2}\right)$.

We use the calibrated model to investigate the extent to which the households' deductible choices would change if premiums were experience rated. In particular, we examine how the distribution of model-predicted deductible choices changes when we counterfactually move from prior premiums (i.e., premiums generated using non-experience rated base prices, $\bar{p}_{i k}$ ) to uniline premiums and multiline premiums (i.e., premiums generated using uniline experienced rated base prices, $\bar{p}_{i k}^{U L}$, and multiline experienced rated base prices, $\left.\bar{p}_{i k}^{M L}\right)$. In each case, we assume that households believe their claim probability is $\mu_{i}=1-\exp \left(-\widehat{\theta}_{i}\right)$.

Table A9 presents the results. In addition to displaying the distributions, the table reports the percentage of policies in which the deductible choice changes when we move from prior premiums to uniline and multiline premiums, as well as the resulting (absolute) changes in coverage. The main takeaway here is the same as it is under the expected utility model - the response of deductible choices to experience rating would be substantial. When we move from prior premiums to uniline premiums, the deductible choice changes in 5 percent of auto collision policies, 14 percent of auto comprehensive policies, and 12 percent of home policies, resulting in average changes in coverage of $\$ 262, \$ 144$, and $\$ 336$, respectively, among policies with a change, and of $\$ 14, \$ 21$, and $\$ 39$, respectively, among all policies. When we move from prior premiums to multiline premiums, the deductible choice changes in 7 percent of auto collision policies, 25 percent of auto comprehensive policies, and 13 percent of home policies, resulting in average changes in coverage of $\$ 262, \$ 149$, and $\$ 337$, respectively, among policies with a change and of $\$ 18, \$ 38$, and $\$ 43$, respectively, among all policies. 
Figure 1: Kernel density of $\eta_{i t k}$.

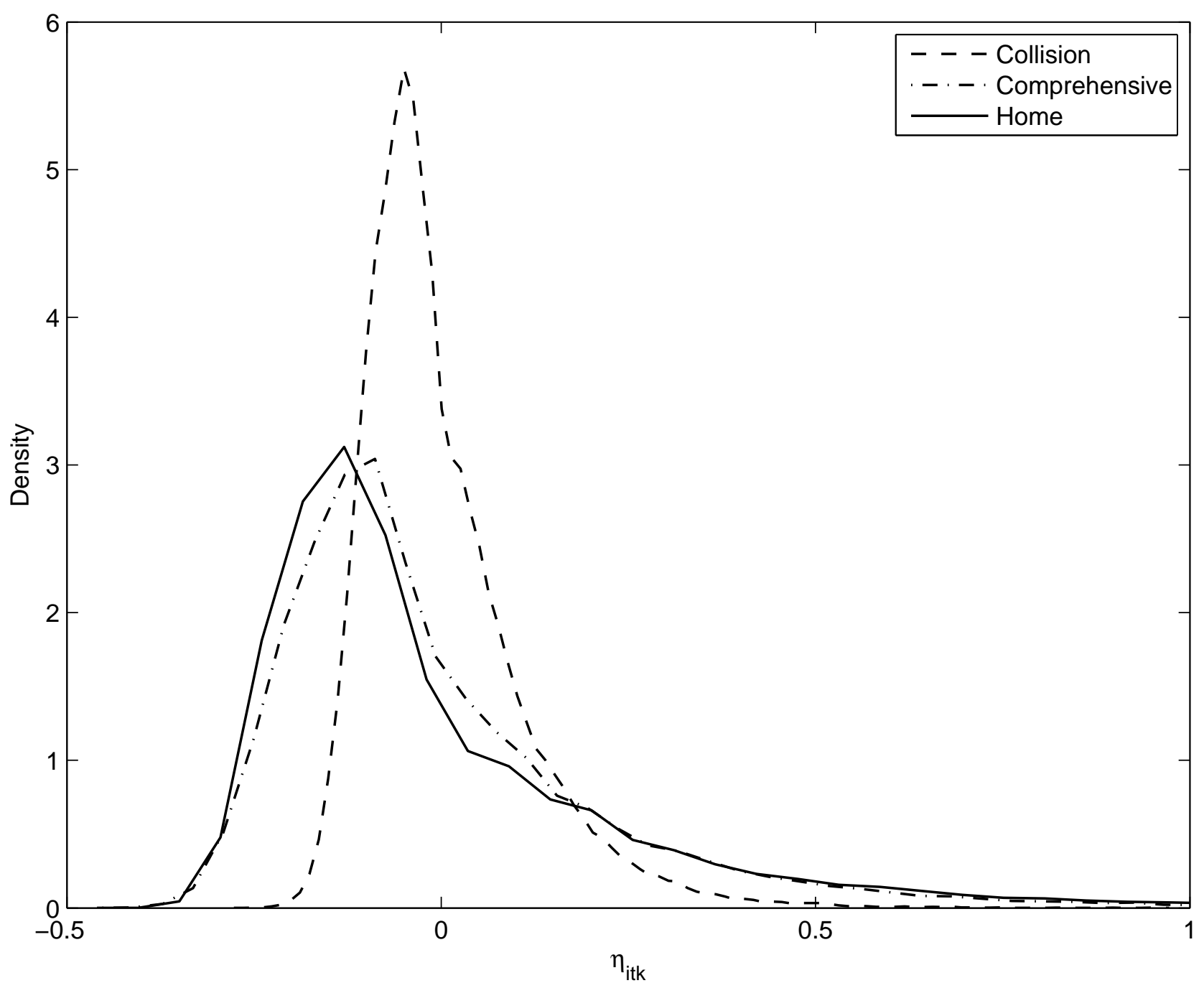


Figure 2: Kernel density of $\zeta_{i t k}$.

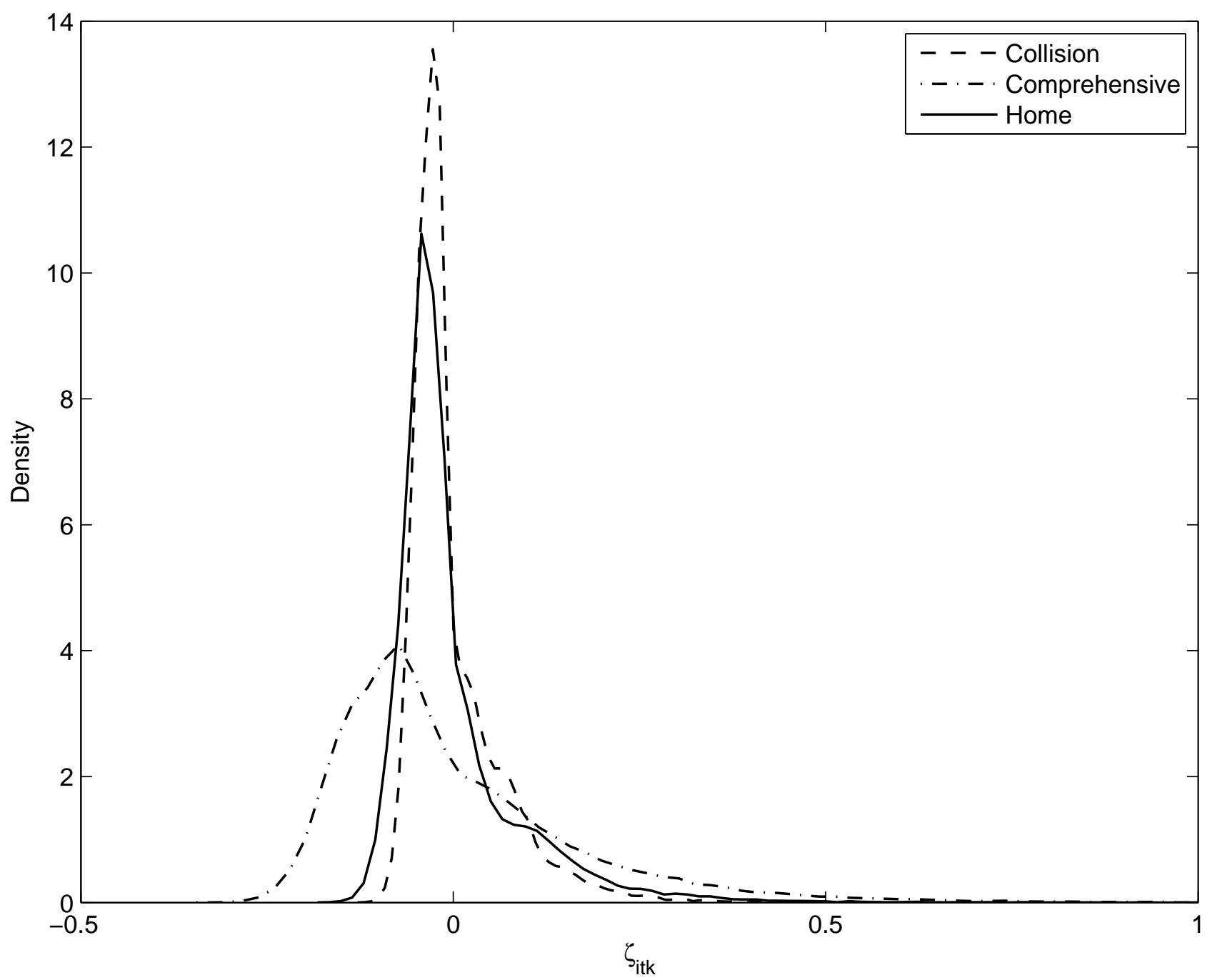


Table 1: Association Parameter Estimates Tricoverage Sample (294,917 household-years)

\begin{tabular}{lccc}
\hline \hline & & \multicolumn{2}{c}{95 percent } \\
& Estimate & confidence interval \\
\hline Variances: & & & \\
Auto collision & 0.107 & 0.065 & 0.149 \\
Auto comprehensive & 0.399 & 0.221 & 0.577 \\
Home & 0.405 & 0.383 & 0.428 \\
\hline Covariances: & & & \\
Auto collision and auto comprehensive & 0.137 & 0.101 & 0.173 \\
Auto collision and home & 0.061 & 0.022 & 0.099 \\
Auto comprehensive and home & 0.225 & 0.179 & 0.271 \\
\hline Correlations: & & & \\
Auto collision and auto comprehensive & 0.663 & 0.399 & 0.926 \\
Auto collision and home & 0.293 & 0.099 & 0.486 \\
Auto comprehensive and home & 0.559 & 0.389 & 0.729 \\
\hline \hline
\end{tabular}


Table 2: Descriptive Statistics for $\eta=(\theta-\lambda) / \lambda$

Tricoverage Sample $(294,917$ household-years $)$

\begin{tabular}{|c|c|c|c|c|c|c|c|c|c|}
\hline \multirow[b]{2}{*}{ Coverage } & \multirow{2}{*}{$\begin{array}{l}\text { Prior } \\
\text { claim } \\
\text { rates }\end{array}$} & \multicolumn{4}{|c|}{$\eta<0$} & \multicolumn{4}{|c|}{$\eta>0$} \\
\hline & & $O b s$ & Mean & $\begin{array}{c}\text { 10th } \\
\text { percentile }\end{array}$ & $\begin{array}{c}25 \text { th } \\
\text { percentile }\end{array}$ & $O b s$ & Mean & $\begin{array}{c}75 \text { th } \\
\text { percentile }\end{array}$ & $\begin{array}{c}\text { 90th } \\
\text { percentile }\end{array}$ \\
\hline \multirow{4}{*}{ Auto collision } & All & 180,909 & -0.066 & -0.121 & -0.093 & 114,008 & 0.101 & 0.140 & 0.227 \\
\hline & Low & 46,914 & -0.055 & -0.100 & -0.080 & 26,815 & 0.094 & 0.129 & 0.213 \\
\hline & Medium & 89,988 & -0.066 & -0.120 & -0.094 & 57,471 & 0.100 & 0.139 & 0.223 \\
\hline & High & 44,007 & -0.078 & -0.143 & -0.110 & 29,722 & 0.110 & 0.152 & 0.249 \\
\hline \multirow{4}{*}{$\begin{array}{c}\text { Auto } \\
\text { Comprehensive }\end{array}$} & All & 188,792 & -0.132 & -0.236 & -0.186 & 106,125 & 0.231 & 0.310 & 0.531 \\
\hline & Low & 47,384 & -0.111 & -0.198 & -0.157 & 26,345 & 0.198 & 0.270 & 0.455 \\
\hline & Medium & 94,742 & -0.131 & -0.233 & -0.186 & 52,717 & 0.231 & 0.307 & 0.533 \\
\hline & High & 46,666 & -0.155 & -0.273 & -0.220 & 27,063 & 0.264 & 0.353 & 0.593 \\
\hline \multirow{4}{*}{ Home } & All & 196,205 & -0.142 & -0.241 & -0.198 & 98,712 & 0.280 & 0.367 & 0.646 \\
\hline & Low & 51,136 & -0.120 & -0.207 & -0.166 & 22,593 & 0.273 & 0.350 & 0.630 \\
\hline & Medium & 97,208 & -0.146 & -0.238 & -0.200 & 50,251 & 0.277 & 0.365 & 0.642 \\
\hline & High & 47,861 & -0.159 & -0.271 & -0.227 & 25,868 & 0.292 & 0.390 & 0.663 \\
\hline
\end{tabular}

Notes: A prior claim rate is "low" if it is in the bottom quartile and "high" if it is in the top quartile. It is "medium" otherwise. In the tricoverage sample, the respective low and high cutoffs are 0.078 and 0.127 in auto collision, 0.016 and 0.044 in auto comprehensive, and 0.054 and 0.096 in home. 
Table 3: Descriptive Statistics for $\zeta=(\theta-\vartheta) / \vartheta$

Tricoverage Sample (294,917 household-years)

\begin{tabular}{|c|c|c|c|c|c|c|c|c|c|}
\hline \multirow[b]{2}{*}{ Coverage } & \multirow{2}{*}{$\begin{array}{l}\text { Prior } \\
\text { claim } \\
\text { rates }\end{array}$} & \multicolumn{4}{|c|}{$\zeta<0$} & \multicolumn{4}{|c|}{$\zeta>0$} \\
\hline & & $O b s$ & Mean & $\begin{array}{c}\text { 10th } \\
\text { percentile }\end{array}$ & $\begin{array}{c}25 \text { th } \\
\text { percentile }\end{array}$ & $O b s$ & Mean & $\begin{array}{c}75 \text { th } \\
\text { percentile }\end{array}$ & $\begin{array}{c}\text { 90th } \\
\text { percentile }\end{array}$ \\
\hline \multirow{4}{*}{ Auto collision } & All & 198,557 & -0.034 & -0.059 & -0.046 & 96,360 & 0.069 & 0.093 & 0.153 \\
\hline & Low & 51,883 & -0.030 & -0.052 & -0.041 & 21,846 & 0.067 & 0.092 & 0.148 \\
\hline & Medium & 99,078 & -0.034 & -0.059 & -0.047 & 48,381 & 0.069 & 0.093 & 0.153 \\
\hline & High & 47,596 & -0.038 & -0.065 & -0.052 & 26,133 & 0.070 & 0.095 & 0.157 \\
\hline \multirow{4}{*}{$\begin{array}{c}\text { Auto } \\
\text { Comprehensive }\end{array}$} & All & 183,662 & -0.098 & -0.173 & -0.139 & 111,255 & 0.157 & 0.217 & 0.357 \\
\hline & Low & 46,521 & -0.095 & -0.171 & -0.135 & 27,208 & 0.163 & 0.224 & 0.370 \\
\hline & Medium & 91,981 & -0.099 & -0.175 & -0.140 & 55,478 & 0.159 & 0.220 & 0.359 \\
\hline & High & 45,160 & -0.098 & -0.173 & -0.139 & 28,569 & 0.149 & 0.207 & 0.334 \\
\hline \multirow{4}{*}{ Home } & All & 202,137 & -0.044 & -0.079 & -0.059 & 92,780 & 0.093 & 0.132 & 0.211 \\
\hline & Low & 51,610 & -0.040 & -0.074 & -0.055 & 22,119 & 0.093 & 0.135 & 0.213 \\
\hline & Medium & 101,001 & -0.046 & -0.082 & -0.062 & 46,458 & 0.095 & 0.134 & 0.214 \\
\hline & High & 49,526 & -0.044 & -0.080 & -0.059 & 24,203 & 0.090 & 0.126 & 0.203 \\
\hline
\end{tabular}

Notes: A prior claim rate is "low" if it is in the bottom quartile and "high" if it is in the top quartile. It is "medium" otherwise. In the tricoverage sample, the respective low and high cutoffs are 0.078 and 0.127 in auto collision, 0.016 and 0.044 in auto comprehensive, and 0.054 and 0.096 in home. 
Table 4: Summary of Bonus-Malus Coefficients

Tricoverage Sample (294,917 household-years)

\begin{tabular}{lccccccc}
\hline \hline & \multicolumn{3}{c}{ Univariate } & & \multicolumn{3}{c}{ Multivariate } \\
\cline { 2 - 3 } \cline { 7 - 8 } & Coll & Comp & Home & & Coll & Comp & Home \\
\hline Mean & 1.00 & 1.00 & 1.00 & & 1.00 & 1.00 & 1.00 \\
Standard deviation & 0.08 & 0.17 & 0.26 & & 0.11 & 0.24 & 0.27 \\
\hline 5th percentile & 0.91 & 0.86 & 0.79 & & 0.87 & 0.75 & 0.75 \\
10th percentile & 0.92 & 0.89 & 0.81 & & 0.89 & 0.78 & 0.78 \\
25th percentile & 0.94 & 0.92 & 0.85 & & 0.93 & 0.85 & 0.83 \\
Median & 0.97 & 0.96 & 0.91 & & 0.97 & 0.93 & 0.91 \\
75th percentile & 1.04 & 0.98 & 1.08 & & 1.05 & 1.08 & 1.08 \\
90th percentile & 1.11 & 1.23 & 1.29 & & 1.14 & 1.28 & 1.32 \\
95th percentile & 1.16 & 1.32 & 1.50 & & 1.20 & 1.45 & 1.53 \\
\hline \hline
\end{tabular}


Table 5: Response of Deductible Choices to Experience Rating Tricoverage Sample (62,425 policies)

Panel A: Auto collision

\begin{tabular}{cccc} 
Deductible choice & $\begin{array}{c}\text { Prior } \\
\text { premiums }\end{array}$ & $\begin{array}{c}\text { Uniline } \\
\text { premiums }\end{array}$ & $\begin{array}{c}\text { Multiline } \\
\text { premiums }\end{array}$ \\
\hline$\$ 100$ & 2,374 & 2,389 & 2,409 \\
$\$ 200$ & 0 & 0 & 0 \\
$\$ 250$ & 34,564 & 34,723 & 34,751 \\
$\$ 500$ & 24,004 & 23,872 & 23,808 \\
Policies with change in deductible (percent) & 1,483 & 1,441 & 1,457 \\
Aggregate changes in coverage (dollars), gross (absolute value) & - & 5.3 & 6.8 \\
Per policy change in coverage (dollars), policies with change & - & 246.99 & 246.86 \\
Per policy change in coverage (dollars), all policies & - & 13.15 & 16.76 \\
\hline
\end{tabular}

Panel B: Auto comprehensive

\begin{tabular}{cccc} 
Deductible choice & $\begin{array}{c}\text { Prior } \\
\text { premiums }\end{array}$ & $\begin{array}{c}\text { Uniline } \\
\text { premiums }\end{array}$ & $\begin{array}{c}\text { Multiline } \\
\text { premiums }\end{array}$ \\
\hline$\$ 50$ & 7 & 4 & 4 \\
$\$ 100$ & 3,633 & 3,723 & 3,633 \\
$\$ 200$ & 10,444 & 10,643 & 10,822 \\
$\$ 250$ & 15,144 & 15,359 & 15,636 \\
$\$ 500$ & 27,979 & 27,530 & 27,276 \\
$\$ 1,000$ & 5,218 & 5,166 & 5,054 \\
Policies with change in deductible (percent) & - & 12.4 & 20.9 \\
Aggregate changes in coverage (dollars), gross & - & $1,276,800$ & $2,348,350$ \\
Per policy change in coverage (dollars), policies with change & - & 164.47 & 179.91 \\
Per policy change in coverage (dollars), all policies & - & 20.45 & 37.62 \\
\hline
\end{tabular}

Panel C: Home

\begin{tabular}{cccc} 
Deductible choice & $\begin{array}{c}\text { Prior } \\
\text { premiums }\end{array}$ & $\begin{array}{c}\text { Uniline } \\
\text { premiums }\end{array}$ & $\begin{array}{c}\text { Multiline } \\
\text { premiums }\end{array}$ \\
\hline$\$ 100$ & 85 & 69 & 70 \\
$\$ 250$ & 31,871 & 32,851 & 32,993 \\
$\$ 500$ & 18,440 & 17,972 & 17,847 \\
$\$ 1,000$ & 11,632 & 11,160 & 11,139 \\
$\$ 2,500$ & 318 & 304 & 303 \\
$\$ 5,000$ & 79 & 69 & 73 \\
Policies with change in deductible (percent) & - & 13.5 & 14.7 \\
Aggregate changes in coverage (dollars), gross & - & $2,902,500$ & $3,187,200$ \\
Per policy change in coverage (dollars), policies with change & - & 343.45 & 347.45 \\
Per policy change in coverage (dollars), all policies & - & 46.50 & 51.06 \\
\hline \hline
\end{tabular}


Table A1: Descriptive Statistics

Tricoverage Sample (294,917 household-years)

\begin{tabular}{|c|c|c|c|c|}
\hline & Mean & Std Dev & Min & Max \\
\hline \multicolumn{5}{|l|}{ Auto: } \\
\hline Driver 1 age (years) & 56.10 & 14.70 & 19 & 99 \\
\hline Driver 1 female & 0.33 & 0.47 & 0 & 1 \\
\hline Driver 1 single & 0.22 & 0.41 & 0 & 1 \\
\hline Driver 1 married & 0.63 & 0.48 & 0 & 1 \\
\hline Driver 1 insurance score & 789.51 & 106.50 & 297 & 996 \\
\hline Driver 2 & 0.48 & 0.50 & 0 & 1 \\
\hline Driver 2 age (years) & 50.28 & 12.93 & 16 & 94 \\
\hline Driver 2 female & 0.91 & 0.28 & 0 & 1 \\
\hline Driver $3+$ & 0.04 & 0.21 & 0 & 1 \\
\hline Young driver & 0.01 & 0.10 & 0 & 1 \\
\hline Vehicle 1 age (years) & 4.43 & 3.59 & -1 & 46 \\
\hline Vehicle 1 personal use & 0.47 & 0.50 & 0 & 1 \\
\hline Vehicle 1 passive restraint & 0.99 & 0.10 & 0 & 1 \\
\hline Vehicle 1 anti-theft & 0.57 & 0.49 & 0 & 1 \\
\hline Vehicle 1 anti-lock brakes & 0.79 & 0.41 & 0 & 1 \\
\hline Vehicle 2 & 0.53 & 0.50 & 0 & 1 \\
\hline Vehicle 2 age (years) & 5.94 & 5.53 & -1 & 83 \\
\hline Vehicle 2 personal use & 0.55 & 0.50 & 0 & 1 \\
\hline Vehicle 2 passive restraint & 0.94 & 0.24 & 0 & 1 \\
\hline Vehicle 2 anti-theft & 0.46 & 0.50 & 0 & 1 \\
\hline Vehicle 2 anti-lock brakes & 0.70 & 0.46 & 0 & 1 \\
\hline Vehicle $3+$ & 0.05 & 0.22 & 0 & 1 \\
\hline \multicolumn{5}{|l|}{ Home: } \\
\hline Home age (years) & 45.05 & 27.20 & 0 & 206 \\
\hline Insured value (thousands of dollars) & 153.31 & 75.63 & 1 & 3,250 \\
\hline Farm or business & 0.02 & 0.15 & 0 & 1 \\
\hline Primary residence & 1.00 & 0.04 & 0 & 1 \\
\hline Owner occupied & 0.98 & 0.14 & 0 & 1 \\
\hline Number of families & 1.16 & 1.89 & 1 & 99 \\
\hline Masonry construction & 0.07 & 0.25 & 0 & 1 \\
\hline Distance to fire hydrant (feet) & 401.83 & 514.82 & 0 & 30,000 \\
\hline Alarm or other protection & 0.95 & 0.22 & 0 & 1 \\
\hline
\end{tabular}

Note: Insurance score is based on information contained in credit reports. 
Table A2: Claims

Tricoverage Sample (294,917 household-years)

\begin{tabular}{|c|c|c|c|c|c|c|}
\hline \multirow[b]{2}{*}{ Count } & \multicolumn{2}{|c|}{ Auto collision } & \multicolumn{2}{|c|}{ Auto comprehensive } & \multicolumn{2}{|c|}{ Home } \\
\hline & Frequency & Percent & Frequency & Percent & Frequency & Percent \\
\hline 0 & 265,692 & 90 & 285,923 & 97 & 273,984 & 93 \\
\hline 1 & 27,186 & 9 & 8,495 & 3 & 18,886 & 6 \\
\hline 2 & 1,890 & 1 & 467 & 0 & 1,872 & 1 \\
\hline 3 & 140 & 0 & 30 & 0 & 159 & 0 \\
\hline 4 & 6 & - & 0 & - & 12 & - \\
\hline 5 & 3 & - & 2 & - & 2 & - \\
\hline 6 & & & & & 2 & - \\
\hline
\end{tabular}

Note: Dash indicates less than 0.01 percent. 
Table A3: Premiums

Tricoverage Sample (294,917 household-years)

\begin{tabular}{|c|c|c|c|}
\hline & \multicolumn{3}{|c|}{ Auto } \\
\hline & Auto collision & comprehensive & Home \\
\hline Mean & 200 & 127 & 548 \\
\hline Standard deviation & 104 & 70 & 309 \\
\hline Minimum & 20 & 6 & 50 \\
\hline 1st percentile & 60 & 34 & 204 \\
\hline 5th percentile & 82 & 48 & 265 \\
\hline 10th percentile & 97 & 58 & 296 \\
\hline 25th percentile & 129 & 81 & 359 \\
\hline Median & 178 & 113 & 466 \\
\hline 75th percentile & 243 & 157 & 638 \\
\hline 90th percentile & 327 & 210 & 891 \\
\hline 95th percentile & 393 & 250 & 1,110 \\
\hline 99th percentile & 560 & 358 & 1,683 \\
\hline Maximum & 2,520 & 2,524 & 10,224 \\
\hline
\end{tabular}

Note: Amounts in dollars. 
Table A4: Deductibles

Tricoverage Sample (294,917 household-years)

\begin{tabular}{|c|c|c|c|c|c|c|}
\hline \multirow[b]{2}{*}{ Deductible } & \multicolumn{2}{|c|}{ Auto collision } & \multicolumn{2}{|c|}{ Auto comprehensive } & \multicolumn{2}{|c|}{ Home } \\
\hline & Frequency & Percent & Frequency & Percent & Frequency & Percent \\
\hline$\$ 50$ & - & - & 34,007 & 12 & - & - \\
\hline$\$ 100$ & 7,846 & 3 & 18,502 & 6 & 11,577 & 4 \\
\hline$\$ 200$ & 65,672 & 22 & 128,599 & 44 & - & - \\
\hline$\$ 250$ & 51,644 & 18 & 31,556 & 11 & 197,100 & 67 \\
\hline$\$ 500$ & 159,702 & 54 & 78,098 & 26 & 70,567 & 24 \\
\hline$\$ 1,000$ & 10,053 & 3 & 4,155 & 1 & 14,537 & 5 \\
\hline$\$ 2,500$ & - & - & - & - & 1,044 & 0 \\
\hline$\$ 5,000$ & - & - & - & - & 92 & 0 \\
\hline
\end{tabular}

Note: Dash indicates deductible option not available. 
Table A5: Regression Parameter Estimates - Auto

Tricoverage Sample (294,917 household-years)

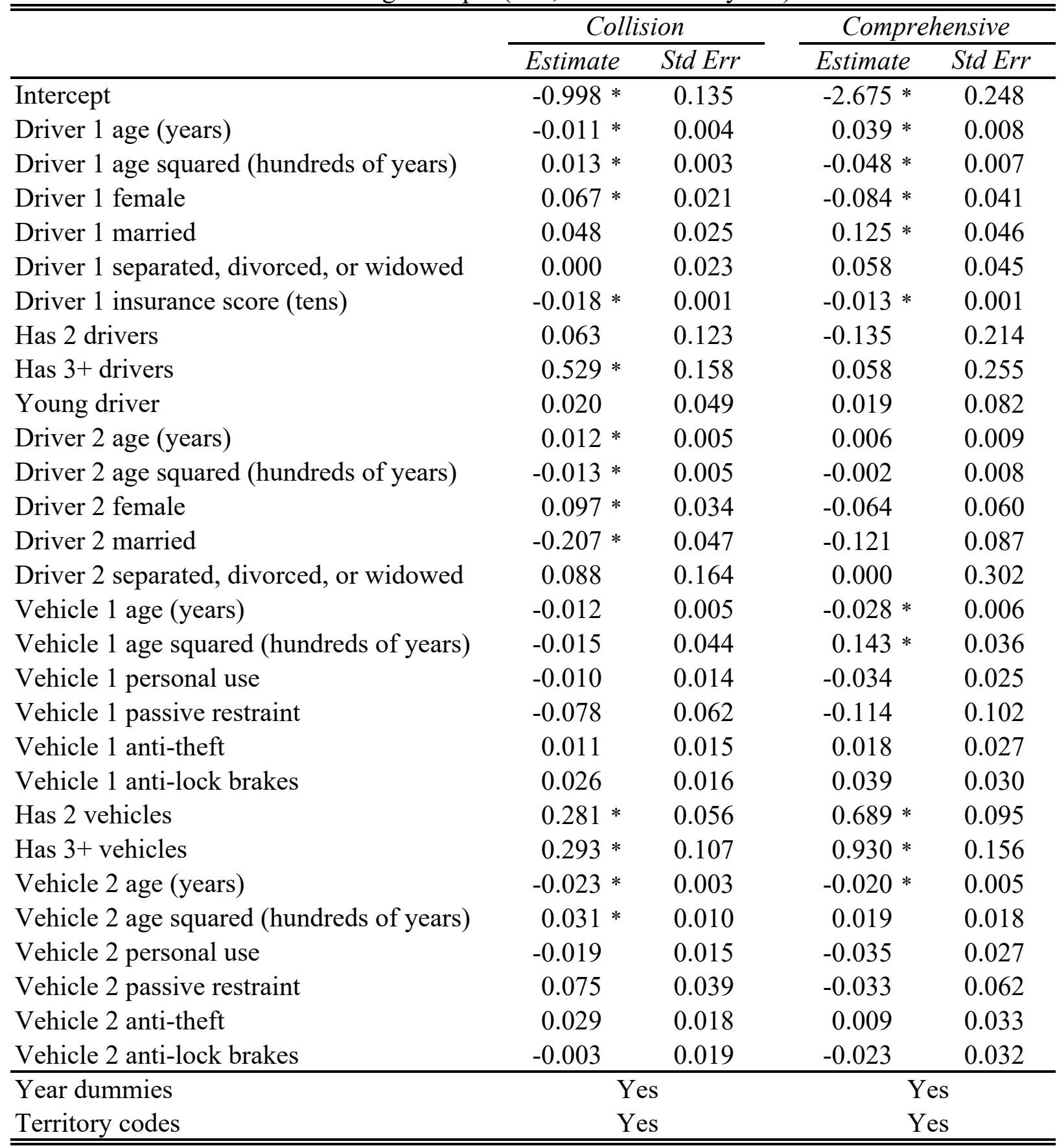

Notes: Insurance score is based on information contained in credit reports. Territory codes indicate rating territories, which are based on actuarial risk factors, such as traffic and weather patterns, population demographics, wildlife density, and the cost of goods and services.

* Significant at the 5 percent level. 
Table A6: Regression Parameter Estimates - Home Tricoverage Sample (294,917 household-years)

\begin{tabular}{lcc}
\hline \hline & Estimate & Std Err \\
\hline Intercept & $-1.968 *$ & 0.250 \\
Insurance score (tens) & $-0.018 *$ & 0.001 \\
Home age (years) & $0.003 *$ & 0.001 \\
Home age squared (years) & 0.000 & 0.000 \\
Insured value (tens of thousands of dollars) & $0.015 *$ & 0.001 \\
Farm or business & $0.098 *$ & 0.047 \\
Primary residence & $0.631 *$ & 0.228 \\
Owner occupied & 0.121 & 0.077 \\
Number of families & -0.011 & 0.007 \\
Masonry construction & 0.048 & 0.029 \\
Distance to fire hydrant (feet) & 0.001 & 0.001 \\
Alarm or other protection & 0.019 & 0.036 \\
\hline Year dummies & \multicolumn{2}{c}{ Yes } \\
Territory codes & \multicolumn{2}{c}{ Yes } \\
Protection classes & \multicolumn{2}{c}{ Ces } \\
\hline \hline
\end{tabular}

Notes: Insurance score is based on information contained in credit reports. Territory codes indicate rating territories, which are based on actuarial risk factors, such as traffic and weather patterns, population demographics, wildlife density, and the cost of goods and services. Protection classes gauge the effectiveness of local fire protection and building codes.

* Significant at the 5 percent level. 
Table A7: Association Parameter Estimates - Alternative Samples

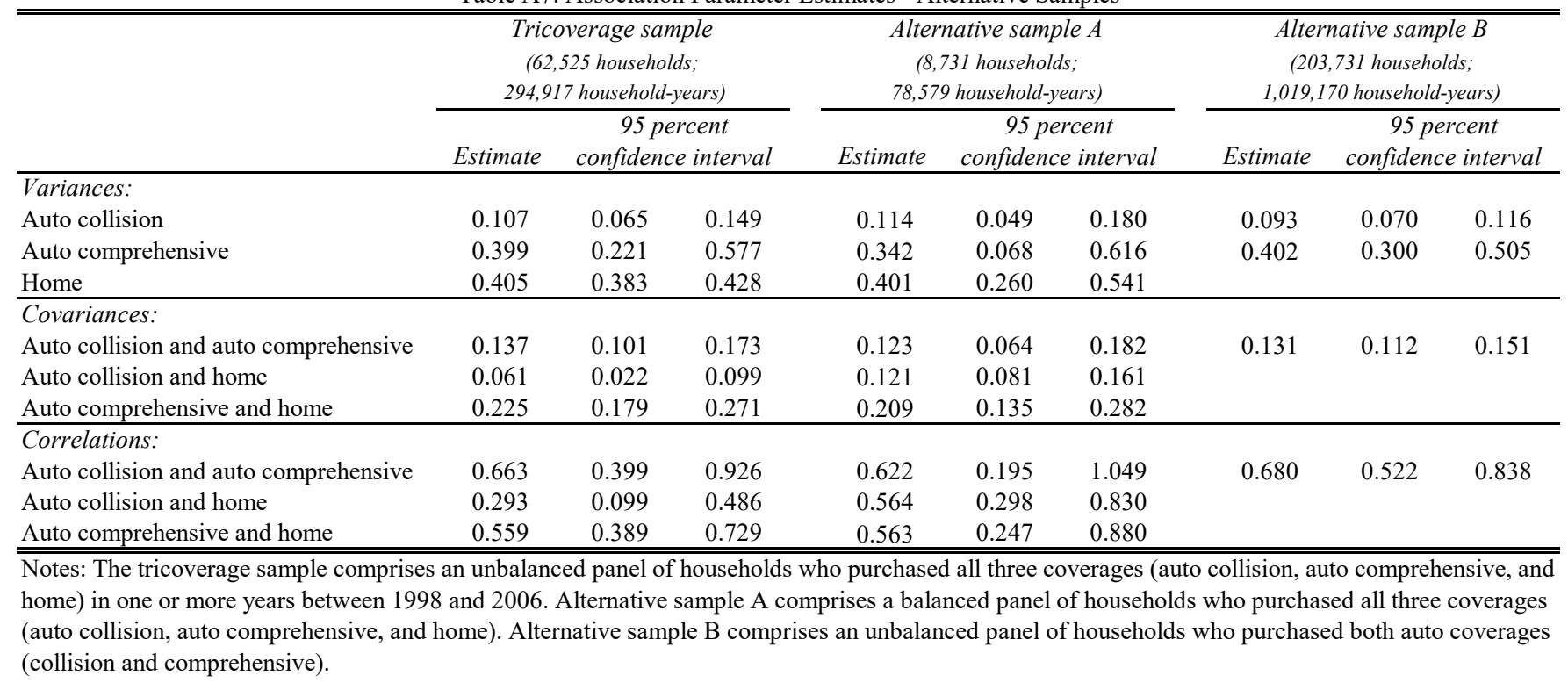


Table A8: Association Parameter Estimates - Low and High Deductible Households

\begin{tabular}{|c|c|c|c|c|c|c|c|c|c|}
\hline \multirow{3}{*}{ Variances: } & \multicolumn{3}{|c|}{$\begin{array}{l}\text { Tricoverage sample } \\
(62,425 \text { households; } \\
294,917 \text { household-years })\end{array}$} & \multicolumn{3}{|c|}{$\begin{array}{c}\text { All deductibles } \leq \$ 250 \\
(22,072 \text { households; } \\
120,213 \text { household-years })\end{array}$} & \multicolumn{3}{|c|}{$\begin{array}{c}\text { Any deductible }>\$ 250 \\
(40,353 \text { households; } \\
\text { 174,704 household-years })\end{array}$} \\
\hline & \multirow[t]{2}{*}{ Estimate } & \multicolumn{2}{|c|}{$\begin{array}{c}95 \text { percent } \\
\text { confidence interval }\end{array}$} & \multirow[t]{2}{*}{ Estimate } & \multicolumn{2}{|c|}{$\begin{array}{c}95 \text { percent } \\
\text { confidence interval }\end{array}$} & \multirow[t]{2}{*}{ Estimate } & \multicolumn{2}{|c|}{$\begin{array}{c}95 \text { percent } \\
\text { confidence interval }\end{array}$} \\
\hline & & & & & & & & & \\
\hline Auto collision & 0.107 & 0.065 & 0.149 & 0.094 & 0.038 & 0.150 & 0.108 & 0.051 & 0.166 \\
\hline Auto comprehensive & 0.399 & 0.221 & 0.577 & 0.337 & 0.086 & 0.587 & 0.450 & 0.201 & 0.698 \\
\hline Home & 0.405 & 0.383 & 0.428 & 0.388 & 0.281 & 0.496 & 0.246 & 0.038 & 0.454 \\
\hline \multicolumn{10}{|l|}{ Covariances: } \\
\hline Auto collision and auto comprehensive & 0.137 & 0.101 & 0.173 & 0.138 & 0.085 & 0.192 & 0.129 & 0.081 & 0.178 \\
\hline Auto collision and home & 0.061 & 0.022 & 0.099 & 0.088 & 0.055 & 0.120 & 0.058 & 0.021 & 0.094 \\
\hline Auto comprehensive and home & 0.225 & 0.179 & 0.271 & 0.224 & 0.157 & 0.290 & 0.217 & 0.152 & 0.282 \\
\hline \multicolumn{10}{|l|}{ Correlations: } \\
\hline Auto collision and auto comprehensive & 0.663 & 0.399 & 0.926 & 0.776 & 0.300 & 1.252 & 0.586 & 0.272 & 0.900 \\
\hline Auto collision and home & 0.293 & 0.099 & 0.486 & 0.458 & 0.230 & 0.686 & 0.352 & 0.066 & 0.639 \\
\hline Auto comprehensive and home & 0.559 & 0.389 & 0.729 & 0.619 & 0.312 & 0.926 & 0.652 & 0.270 & 1.034 \\
\hline
\end{tabular}


Table A9: Response of Deductible Choices to Experience Rating Tricoverage Sample $(62,425$ policies $)$

\begin{tabular}{|c|c|c|c|c|c|c|}
\hline \multirow[b]{3}{*}{ Deductible choice } & \multicolumn{6}{|c|}{ Panel A: Auto collision } \\
\hline & \multicolumn{3}{|c|}{ Expected utility model } & \multicolumn{3}{|c|}{ Probability distortion model } \\
\hline & $\begin{array}{c}\text { Prior } \\
\text { premiums }\end{array}$ & $\begin{array}{c}\text { Uniline } \\
\text { premiums }\end{array}$ & $\begin{array}{l}\text { Multiline } \\
\text { premiums }\end{array}$ & $\begin{array}{c}\text { Prior } \\
\text { premiums }\end{array}$ & $\begin{array}{c}\text { Uniline } \\
\text { premiums }\end{array}$ & $\begin{array}{l}\text { Multiline } \\
\text { premiums }\end{array}$ \\
\hline$\$ 100$ & 2,374 & 2,389 & 2,409 & 1,194 & 1,273 & 1,279 \\
\hline$\$ 200$ & 0 & 0 & 0 & 0 & 0 & 0 \\
\hline$\$ 250$ & 34,564 & 34,723 & 34,751 & 29,379 & 29,428 & 29,524 \\
\hline$\$ 500$ & 24,004 & 23,872 & 23,808 & 30,194 & 30,018 & 29,925 \\
\hline$\$ 1,000$ & 1,483 & 1,441 & 1,457 & 1,658 & 1,706 & 1,697 \\
\hline Policies with change in deductible (percent) & - & 5.3 & 6.8 & - & 5.3 & 6.8 \\
\hline Aggregate changes in coverage (dollars), gross (absolute value) & - & 820,750 & $1,046,450$ & - & 864,850 & $1,111,950$ \\
\hline Per policy change in coverage (dollars), policies with change & - & 246.99 & 246.86 & - & 262.47 & 261.64 \\
\hline Per policy change in coverage (dollars), all policies & - & 13.15 & 16.76 & - & 13.85 & 17.81 \\
\hline
\end{tabular}

Panel B: Auto comprehensive

\begin{tabular}{|c|c|c|c|c|c|c|}
\hline \multirow[b]{2}{*}{ Deductible choice } & \multicolumn{3}{|c|}{ Expected utility model } & \multicolumn{3}{|c|}{ Probability distortion model } \\
\hline & $\begin{array}{c}\text { Prior } \\
\text { premiums }\end{array}$ & $\begin{array}{c}\text { Uniline } \\
\text { premiums }\end{array}$ & $\begin{array}{l}\text { Multiline } \\
\text { premiums }\end{array}$ & $\begin{array}{c}\text { Prior } \\
\text { premiums }\end{array}$ & $\begin{array}{c}\text { Uniline } \\
\text { premiums }\end{array}$ & $\begin{array}{l}\text { Multiline } \\
\text { premiums }\end{array}$ \\
\hline$\$ 50$ & 7 & 4 & 4 & 20 & 19 & 21 \\
\hline$\$ 100$ & 3,633 & 3,723 & 3,633 & 11,919 & 12,363 & 12,829 \\
\hline$\$ 200$ & 10,444 & 10,643 & 10,822 & 16,976 & 16,949 & 16,862 \\
\hline$\$ 250$ & 15,144 & 15,359 & 15,636 & 12,317 & 12,236 & 12,024 \\
\hline$\$ 500$ & 27,979 & 27,530 & 27,276 & 19,910 & 19,565 & 19,371 \\
\hline$\$ 1,000$ & 5,218 & 5,166 & 5,054 & 1,283 & 1,293 & 1,318 \\
\hline Policies with change in deductible (percent) & - & 12.4 & 20.9 & - & 14.4 & 25.3 \\
\hline Aggregate changes in coverage (dollars), gross & - & $1,276,800$ & $2,348,350$ & - & $1,295,500$ & $2,351,000$ \\
\hline Per policy change in coverage (dollars), policies with change & - & 164.47 & 179.91 & - & 143.70 & 148.69 \\
\hline Per policy change in coverage (dollars), all policies & - & 20.45 & 37.62 & - & 20.75 & 37.66 \\
\hline
\end{tabular}

Panel C: Home

\begin{tabular}{|c|c|c|c|c|c|c|}
\hline \multirow[b]{2}{*}{ Deductible choice } & \multicolumn{3}{|c|}{ Expected utility model } & \multicolumn{3}{|c|}{ Probability distortion model } \\
\hline & $\begin{array}{c}\text { Prior } \\
\text { premiums }\end{array}$ & $\begin{array}{c}\text { Uniline } \\
\text { premiums }\end{array}$ & $\begin{array}{l}\text { Multiline } \\
\text { premiums }\end{array}$ & $\begin{array}{c}\text { Prior } \\
\text { premiums }\end{array}$ & $\begin{array}{c}\text { Uniline } \\
\text { premiums }\end{array}$ & $\begin{array}{l}\text { Multiline } \\
\text { premiums }\end{array}$ \\
\hline$\$ 100$ & 85 & 69 & 70 & 91 & 100 & 98 \\
\hline$\$ 250$ & 31,871 & 32,851 & 32,993 & 40,640 & 40,903 & 40,975 \\
\hline$\$ 500$ & 18,440 & 17,972 & 17,847 & 14,529 & 14,201 & 14,122 \\
\hline$\$ 1,000$ & 11,632 & 11,160 & 11,139 & 7,085 & 7,132 & 7,142 \\
\hline$\$ 2,500$ & 318 & 304 & 303 & 64 & 72 & 69 \\
\hline$\$ 5,000$ & 79 & 69 & 73 & 16 & 17 & 19 \\
\hline Policies with change in deductible (percent) & - & 13.5 & 14.7 & - & 11.6 & 12.8 \\
\hline Aggregate changes in coverage (dollars), gross & - & $2,902,500$ & $3,187,200$ & - & $2,437,850$ & $2,692,850$ \\
\hline Per policy change in coverage (dollars), policies with change & - & 343.45 & 347.45 & - & 335.56 & 337.49 \\
\hline Per policy change in coverage (dollars), all policies & - & 46.50 & 51.06 & - & 39.05 & 43.14 \\
\hline
\end{tabular}

